Article

\title{
Simultaneous Quantitation of Free Amino Acids, Nucleosides and Nucleobases in Sipunculus nudus by Ultra-High Performance Liquid Chromatography with Triple Quadrupole Mass Spectrometry
}

\author{
Yahui Ge ${ }^{1}$, Yuping Tang ${ }^{1, *}$, Sheng Guo ${ }^{1}$, Xin Liu ${ }^{1}$, Zhenhua Zhu ${ }^{1}$, Lili Zhang ${ }^{1}$, Pei Liu ${ }^{1}$, \\ Shaoxiong Ding ${ }^{2}$, Xiangzhi Lin ${ }^{3}$, Rurong Lin $^{3}$ and Jin-ao Duan ${ }^{1}$ \\ 1 Jiangsu Collaborative Innovation Center of Chinese Medicinal Resources Industrialization, and National \\ and Local Collaborative Engineering Center of Chinese Medicinal Resources Industrialization and Formulae \\ Innovative Medicine, Nanjing University of Chinese Medicine, Nanjing 210023, China; \\ geyahui_jsnt@163.com (Y.G.); gsh916@gmail.com (S.G.); liuxin_njutcm@163.com (X.L.); \\ 18913133908@163.com (Z.Z.); lizhang2020@126.com (L.Z.); peiliu1981@126.com (P.L.); \\ dja@njucm.edu.cn (J.D.) \\ 2 College of Ocean and Environment, Xiamen University, Xiamen 361005, China; sxding@xmu.edu.cn \\ 3 Third Institute of Oceanography, State Oceanic Administration, Xiamen 361005, China; \\ xzlin@tio.org.cn (X.L.); linrulong@yahoo.com (R.L.) \\ * Correspondence: yupingtang@njucm.edu.cn; Tel./Fax: +86-25-8581-1695
}

Academic Editor: Derek J. McPhee

Received: 11 January 2016 ; Accepted: 23 March 2016 ; Published: 25 March 2016

\begin{abstract}
To evaluate the nutritional and functional value of Sipunculus nudus, a rapid, simple and sensitive analytical method was developed using ultra-high performance liquid chromatography coupled with a triple quadrupole mass detection in multiple-reaction monitoring mode for the simultaneous quantitative determination of 25 free amino acids and 16 nucleosides and nucleobases in S. nudus within $20 \mathrm{~min}$, which was confirmed to be reproducible and accurate. The limits of detection (LODs) and quantification (LOQs) were between $0.003-0.229 \mu \mathrm{g} / \mathrm{mL}$ and $0.008-0.763 \mu \mathrm{g} / \mathrm{mL}$ for the 41 analytes, respectively. The established method was applied to analyze 19 batches of S. nudus samples from four habitats with two different processing methods. The results showed that $S$. nudus contained a variety of free amino acids, nucleosides and nucleobases in sufficient quantity and reasonable proportion. They also demonstrated that the contents of these compounds in different parts of S. nudus were significantly discriminating, which were in the order: (highest) coelomic fluid > body wall $>$ intestine (lowest). The method is simple and accurate, and could serve as a technical support for establishing quality control of S. nudus and other functional seafoods. Moreover, the research results also laid foundation for further exploitation and development of $S$. nudus.
\end{abstract}

Keywords: UPLC-TQ-MS/MS; Sipunculus nudus; free amino acid; nucleoside; nucleobase

\section{Introduction}

Sipunculus nudus, a Sipunculid species, known as sandworm or haichangzi, has a practically global distribution, except for polar waters. They are unsegmented wormlike animals, comprising two sections namely the trunk (main body) and an introvert (extending and contracting neck-like "feeler") [1]. Called "marine Cordyceps sinensis" by local residents, it has crisp, tender, fresh and sweet taste, can nourish internal organs and clear the internal heat [2], and is ranked as a valuable seafood and senior supplement [3,4]. However, since the 1980s, S. nudus has suffered from predatory and unhindered exploration stimulated by the market price, combined with more and more serious pollution of oceans which have led to a sharp decrease in the availability of the wild resource and 
therefore, more and more attention has been paid to artificial cultivation of the worms [5]. As the technology has developed, the amount of $S$. nudus has increased rapidly and how to utilize the resource reasonably has become a major study topic.

In the last decades, $S$. nudus extract was reported to be rich in a variety of nutritional and functional components consisting of free amino acids, fatty acids, polysaccharides, mineral elements and so on. [6-9]. As we all know, the free amino acids associated with many functional foods such as Ziziphus jujube, royal jelly and Calculus bovis have received considerable attention [10-12]. In recent years, nucleosides and nucleobases have also been proven as important nutritional and functional foodstuffs related to multiple properties such as modulation of the immune response, metabolism, angiocarpy and nervous system as well as antimicrobial and antiviral effects [13]. However, there has been no report about the nucleosides and nucleobases in S. nudus so far. Therefore, in order to compile comprehensive information about the nutritional and functional components in $S$. nudus, we performed a preliminary experiment to detect the nucleosides and nucleobases in $S$. nudus and found such constituents were abundant in $S$. nudus water extract.

In the past years, many researches have been carried out on free amino acids, nucleosides and nucleobases as quality control markers of several functional foods such as Geosaurus, brown seaweeds, royal jelly, Ganoderma lucidum and so on [7,14-17]. However, there are no definite quality control markers for $S$. nudus, although the free amino acids of $S$. nudus have been detected with low sensitivity by visible spectrophotometry after derivatization and complex pretreatment procedures [18]. There have no reports about the contents and proportion of free amino acids, nucleosides and nucleobases in $S$. nudus till now, making it very necessary to develop a fast, convenient and efficient method to precisely measure the amount of these nutritional constituents in $S$. nudus extract, which will be beneficial for expanding its potential value as well as quality control.

In the traditional way, when $S$. nudus is consumed in dishes [2], the internal parts including the intestine and coelomic fluid are usually removed, and then it is cooked alone or with other food materials. The useful constituents in $S$. nudus may be broken down because of high temperature suing during the processing. Therefore, it is necessary to determine the content variation of free amino acids, nucleosides and nucleobases in different parts of $S$. nudus with different methods of sample preparation for the sake of best developing $S$. nudus as a functional seafood.

Ultra-high performance liquid chromatography (UPLC), coupled with mass spectrometry (MS) detection is an important analytical method that has been developed in recent years [19-21]. Due to its efficient separation, high selectivity and high sensitivity, it has been widely used for the quantification and qualitative analysis of active components in biological fluids, medicinal materials and so on [22-26].

In our previous research, the amino acids, nucleosides and nucleobases in another seafood were analyzed by using hydrophilic interaction ultra-performance liquid chromatography coupled with triple quadrupole tandem mass spectrometry [27]. Since the method is simple and accurate, in this study, we also used such a method for simultaneous identification and quantification of 25 free amino acids and 16 nucleosides and nucleobases in different parts of $S$. nudus collected from four habitats with different preparation methods. Then, the data were further handled by a PCA scatter plot to compare the content variation of the samples. The determination of these important components in S. nudus could be vital to quality control as well as tapping its full nutritional and functional value.

\section{Results and Discussion}

\subsection{Sample Preparation Optimization}

To identify as many target components as possible in different parts of $S$. nudus, the extraction solvent (water, aqueous methanol of different concentrations), solvent volume (10, 20, 30, 40, 50, and $60 \mathrm{~mL}$ ), extraction temperature $\left(20,40,60,80,100^{\circ} \mathrm{C}\right)$, extraction method (refluxing and ultrasonication) and extraction time $(10,20,30,40,50$ and $60 \mathrm{~min})$ conditions of the samples from different parts 
(1.0 g, SE, SI, SC) were optimized. All of parameters were investigated by a univariate method using peak area as a measurement. It was found that the best extraction conditions were ultrasonication at $40{ }^{\circ} \mathrm{C}$ for $60 \mathrm{~min}$ with $40 \mathrm{~mL}$ water as solvent. However, to evaluate the free amino acids, nucleosides and nucleobases of $S$. nudus extracted in the traditional way, the extraction method of refluxing for $60 \mathrm{~min}$ was applied to imitate water decoction.

\subsection{UPLC-TQ-MS/MS Conditions Optimization}

In preliminary tests two columns, an Acquity BEH C18 $(100 \mathrm{~mm} \times 2.1 \mathrm{~mm}, 1.7 \mu \mathrm{m})$ and an Acquity BEH Amide $(100 \mathrm{~mm} \times 2.1 \mathrm{~mm}, 1.7 \mu \mathrm{m})$, were compared to obtain chromatograms with better resolution of adjacent peaks, improved peak shape and shortest peak appearance time. On account of the fact that free amino acids, nucleosides and nucleobases are hydrophilic components with high polarity, the results showed that the latter one had a stronger retention ability as well as better resolution under the same mobile phase and other instrument condition circumstances.

As for the mobile phase, as a general rule acetonitrile is known as a polar aprotic solvent with better elution ability, separation selectivity and peak shape compared to methanol, and has been proven to be suitable organic solvent for hydrophilic interaction liquid chromatography with short analysis times. Therefore, a high concentration of acetonitrile was used as organic phase and the concentration was decreased in a gradient. The ammonium acetate and ammonium formate dissolved in acetonitrile are highly volatile, and they can improve the separation of amino acids, nucleosides and nucleobases in the UPLC analysis process [28]. Different mobile phases including independent solutions with different concentration and mixed solutions with different concentration of components were compared. The results showed that a mixed solution containing ammonium formate and ammonium acetate as mobile phase salt additives could increase the sensitivity and improve the peak shapes for these components. The retention times and peak shapes of the compounds were influenced by the different concentrations of ammonium formate and ammonium acetate, consequently, $5 \mathrm{mmol} / \mathrm{L}$ ammonium formate and ammonium acetate in the organic phase and $1 \mathrm{mmol} / \mathrm{L}$ ammonium formate and ammonium acetate in the aqueous phase produced the best shaped peaks in the shortest time. Meanwhile, formic acid is also used to inhibit solute ionization to improve the peak shape so different concentrations of formic acid were added and compared. Eventually, it was determined that the mobile phase should be composed of A $(5 \mathrm{mmol} / \mathrm{L}$ ammonium formate, $5 \mathrm{mmol} / \mathrm{L}$ ammonium acetate and $0.2 \%$ formic acid in aqueous solution $)$ and $B(1 \mathrm{mmol} / \mathrm{L}$ ammonium formate, $1 \mathrm{mmol} / \mathrm{L}$ ammonium acetate and $0.2 \%$ formic acid in acetonitrile) with gradient elution. As regard to flow rate and column temperature, the ranges were both optimized, and the results show that the best mobile phase flow rate was $0.4 \mathrm{~mL} / \mathrm{min}$ and the column temperature was maintained at $35^{\circ} \mathrm{C}$. Analytical chromatograms of the mixed standards and Sample F1 are presented in Figure 1.

For the best MS/MS condition of each analyte, all of the compounds were examined separately in direct infusion mode by a full-scan MS method in both positive and negative ionization modes. The results show that both higher sensitivity and clearer mass spectra were obtained in the positive ion mode compared to the negative ion mode. The free amino acids, nucleosides and nucleobases could combine with $\mathrm{H}^{+}$to give $[\mathrm{M}+\mathrm{H}]^{+}$quasi-molecular ions in $\mathrm{ESI}^{+}$mode. MRM mode was applied in the experiment, and the influence of nucleosides could be minimized because the peak could appear only when the parent and daughter ions were both detected by choosing the appropriate parent and daughter ions. To obtain the best ion pairs, at least two precursor/product ion pairs were chosen for each analyte for quantitative research and the most sensitive and specific ion pairs were selected for the MRM determination. For the nucleosides, $[\mathrm{M}+\mathrm{H}]^{+}$were selected as parent ions and $[\mathrm{M}+\mathrm{H}-\text { deoxyribose }]^{+}$and $[\mathrm{M}+\mathrm{H}-\text { ribose }]^{+}$was selected as daughter ions . As for nucleobases, we have tried to split these compounds before, however, the abundance of product ions for these compounds is too low to be detected by MRM. Consequently, we chose $[\mathrm{M}+\mathrm{H}]^{+}$as both parent and daughter ions for these compounds. For most $\alpha$-amino acids, $[\mathrm{M}+\mathrm{H}-\mathrm{HCOOH}]^{+}$were rearranged to $\left[\mathrm{R}-\mathrm{CH}=\mathrm{NH}_{2}\right]^{+}$as daughter ions. For $\gamma$-aminobutyric acid, $\left[\mathrm{M}+\mathrm{H}-\mathrm{NH}_{3}\right]^{+}$was rearranged to $[\mathrm{R}-\mathrm{CH}-\mathrm{COOH}]^{+}$as daughter ion. 


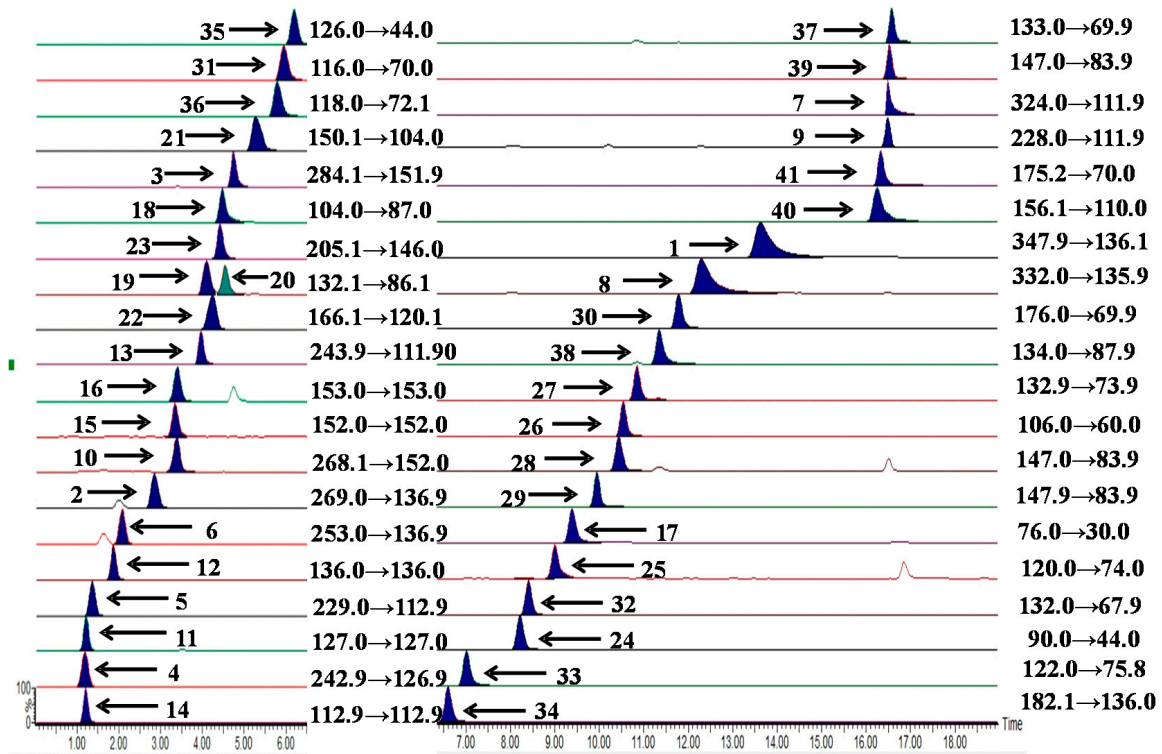

(A)
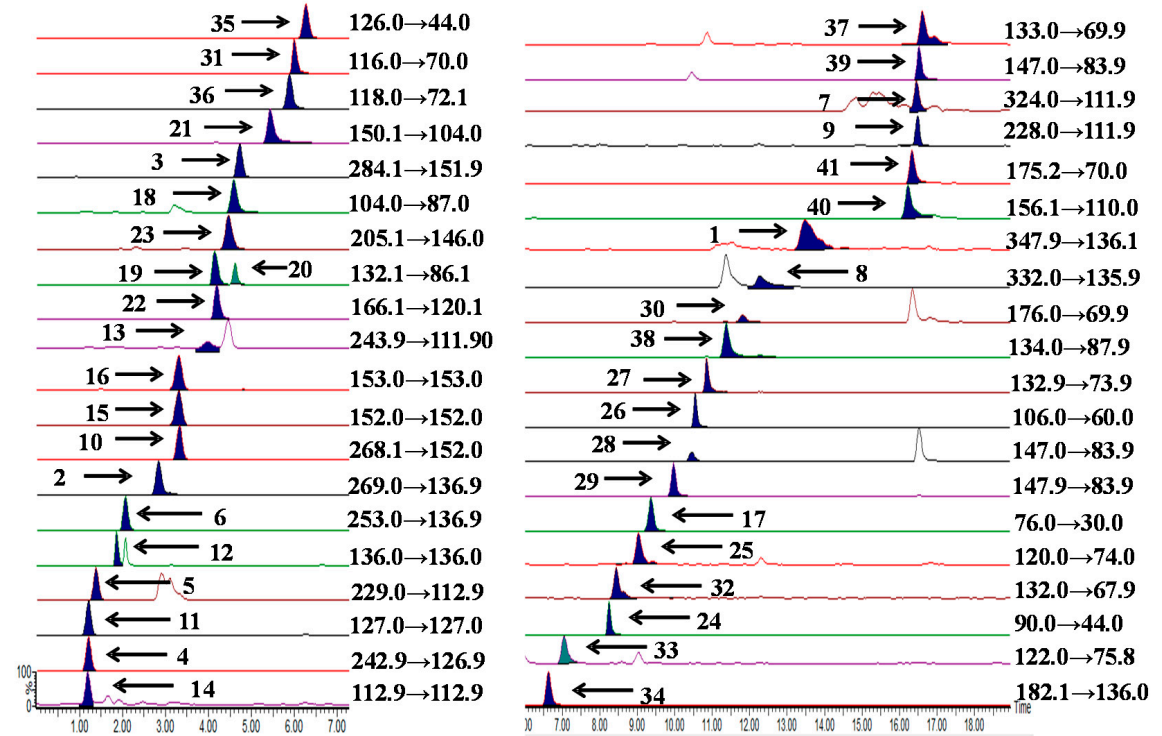

(B)

Figure 1. UHPLC-TQ-MS/MS multiple-reaction monitoring (MRM) chromatograms of the mixed standards (A) and Sample F1 (B). The compound numbers on the chromatograms were adenosine- $5^{\prime}$-monophosphate (1), inosine (2), guanosine (3), thymidine (4), $2^{\prime}$-deoxyuridine (5), $2^{\prime}$-deoxyinosine (6), cytidine-5'-monophosphate (7), 2'-deoxyadenosine-5'-monophosphate (8), $2^{\prime}$-deoxycytidine (9), 2'-deoxyguanosine (10), thymine (11), adenine (12), cytidine (13), uracil (14), guanine (15), xanthine (16), glycine (17), GABA (18), leucine (19), isoleucine (20), methionine (21), phenylalanine (22), tryptophan (23), alanine (24), threonine (25), serine (26), asparagine (27), glutamine (28), glutamate (29), citrulline (30), proline (31), hydroxyproline (32), cysteine (33), tyrosine (34), taurine (35), valine (36), ornithine (37), aspartate (38), lysine (39), histidine (40) and arginine (41), respectively. The peaks of guanine (15) and xanthine (16) were overlapped or embedded.

For glutamine and asparagine, $\left[\mathrm{M}+\mathrm{H}-\mathrm{HCOOH}-\mathrm{NH}_{3}\right]^{+}$were selected as daughter ions. For alkaline amino acids such as arginine, lysine, citrulline and so on, their daughter ions could be affected by the presence of the amino groups of every amino acid (Table 1). Then we optimized cone voltage and collision energy by the function of Intellistart software in the Waters XevoTM TQ MS system. 
Table 1. Precursor/product ion pairs and parameters for MRM of compounds used in this study.

\begin{tabular}{|c|c|c|c|c|c|c|}
\hline NO & Analytes & MW & RT (min) & $\begin{array}{c}\text { MRM } \\
\text { Transitions }\end{array}$ & $\begin{array}{c}\text { Cone } \\
\text { Voltage (V) }\end{array}$ & $\begin{array}{l}\text { Collision } \\
\text { Energy }(\mathrm{eV})\end{array}$ \\
\hline 1 & Adenosine- $5^{\prime}$-monophosphate & 391 & 13.75 & $347.9 \rightarrow 136.1$ & 20 & 20 \\
\hline 2 & Inosine & 268 & 2.96 & $269.0 \rightarrow 136.9$ & 10 & 14 \\
\hline 3 & Guanosine & 283 & 4.88 & $284.1 \rightarrow 151.9$ & 14 & 14 \\
\hline 4 & Thymidine & 242 & 1.34 & $242.9 \rightarrow 126.9$ & 10 & 10 \\
\hline 5 & 2'-Deoxyuridine & 228 & 1.46 & $229.0 \rightarrow 112.9$ & 8 & 10 \\
\hline 6 & $2^{\prime}$-Deoxyinosine & 252 & 2.19 & $253.0 \rightarrow 136.9$ & 22 & 12 \\
\hline 7 & Cytidine-5'-monophosphate & 323 & 16.72 & $324.0 \rightarrow 111.9$ & 16 & 14 \\
\hline 8 & $2^{\prime}$-Deoxyadenosine-5'-mono-phosphate & 329 & 12.53 & $332.0 \rightarrow 135.9$ & 20 & 16 \\
\hline 9 & $2^{\prime}$-Deoxycytidine & 227 & 16.65 & $228.0 \rightarrow 111.9$ & 28 & 10 \\
\hline 10 & 2'-Deoxyguanosine & 267 & 3.55 & $268.1 \rightarrow 152.0$ & 10 & 12 \\
\hline 11 & Thymine & 126 & 1.45 & $127.0 \rightarrow 127.0$ & 30 & 15 \\
\hline 12 & Adenine & 135 & 2.17 & $136.0 \rightarrow 136.0$ & 30 & 20 \\
\hline 13 & Cytidine & 243 & 4.17 & $243.9 \rightarrow 111.9$ & 28 & 10 \\
\hline 14 & Uracil & 112 & 1.20 & $112.9 \rightarrow 112.9$ & 30 & 15 \\
\hline 15 & Guanine & 151 & 3.58 & $152.0 \rightarrow 152.0$ & 30 & 20 \\
\hline 16 & Xanthine & 152 & 3.60 & $153.0 \rightarrow 153.0$ & 30 & 20 \\
\hline 17 & Glycine & 75 & 9.53 & $76.0 \rightarrow 30.0$ & 12 & 6 \\
\hline 18 & GABA & 103 & 4.86 & $104.0 \rightarrow 87.0$ & 16 & 10 \\
\hline 19 & Leucine & 131 & 3.93 & $132.1 \rightarrow 86.1$ & 16 & 10 \\
\hline 20 & Isoleucine & 131 & 4.75 & $132.1 \rightarrow 86.1$ & 16 & 10 \\
\hline 21 & Methionine & 149 & 5.25 & $150.1 \rightarrow 104.0$ & 14 & 10 \\
\hline 22 & Phenylalanine & 165 & 4.32 & $166.1 \rightarrow 120.1$ & 18 & 14 \\
\hline 23 & Tryptophan & 204 & 4.80 & $205.1 \rightarrow 146.0$ & 16 & 18 \\
\hline 24 & Alanine & 89 & 8.46 & $90.0 \rightarrow 44.0$ & 16 & 10 \\
\hline 25 & Threonine & 119 & 9.10 & $120.0 \rightarrow 74.0$ & 38 & 20 \\
\hline 26 & Serine & 105 & 10.65 & $106.0 \rightarrow 60.0$ & 14 & 8 \\
\hline 27 & Asparagine & 132 & 10.81 & $132.9 \rightarrow 73.9$ & 12 & 14 \\
\hline 28 & Glutamine & 146 & 10.60 & $147.0 \rightarrow 83.9$ & 8 & 16 \\
\hline 29 & Glutamate & 147 & 10.11 & $147.9 \rightarrow 83.9$ & 12 & 14 \\
\hline 30 & Citrulline & 175 & 11.98 & $176.0 \rightarrow 69.9$ & 16 & 20 \\
\hline 31 & Proline & 131 & 5.95 & $116.0 \rightarrow 70.0$ & 20 & 10 \\
\hline 32 & Hydroxyproline & 131 & 8.61 & $132.0 \rightarrow 67.9$ & 18 & 16 \\
\hline 33 & Cysteine & 121 & 7.15 & $122.0 \rightarrow 75.8$ & 14 & 17 \\
\hline 34 & Tyrosine & 181 & 6.74 & $182.1 \rightarrow 136.0$ & 16 & 16 \\
\hline 35 & Taurine & 125 & 6.27 & $126.0 \rightarrow 44.0$ & 24 & 14 \\
\hline 36 & Valine & 117 & 5.65 & $118.0 \rightarrow 72.1$ & 12 & 10 \\
\hline 37 & Ornithine & 132 & 17.01 & $133.0 \rightarrow 69.9$ & 14 & 14 \\
\hline 38 & Aspartate & 133 & 11.68 & $132.9 \rightarrow 73.9$ & 12 & 14 \\
\hline 39 & Lysine & 146 & 16.77 & $147.0 \rightarrow 83.9$ & 14 & 14 \\
\hline 40 & Histidine & 155 & 16.53 & $156.1 \rightarrow 110.0$ & 22 & 14 \\
\hline 41 & Arginine & 174 & 16.68 & $175.2 \rightarrow 70.0$ & 22 & 18 \\
\hline
\end{tabular}

The peaks of guanine (15) and xanthine (16) were overlapped.

\subsection{Method Validation}

The established chromatographic method was validated by determining the linearity, LOD, LOQ, intra- and inter-day precisions, repeatability, stability, and recovery. The correlation coefficient values $\left(r^{2}\right)$ showed all calibration curves exhibited good linear regressions $\left(r^{2}>0.9919\right)$ within the determination range of the 41 investigated compounds. The LODs $(\mathrm{S} / \mathrm{N}=3)$ and LOQs $(\mathrm{S} / \mathrm{N}=10)$ of the 41 compounds ranged from $0.003-0.229 \mu \mathrm{g} / \mathrm{mL}$ and $0.008-0.763 \mu \mathrm{g} / \mathrm{mL}$, respectively. The intraday precisions were investigated by determining analytes in six replicates at known concentration during a single day while the interday precisions were determined during three successive day. And the RSDs serve as the measure of precisions. The results showed that RSD values for the intraday precisions were $<3.72 \%$, and for the interday precisions were $<3.42 \%$. The repeatability was evaluated by analyzing six samples processed by the same methods, and the RSDs of the repeatability were $<4.48 \%$. The storage stability of the sample was measured by analyzing the same sample at $0,2,4,8,12,24 \mathrm{~h}$ within one day, and the RSDs of the storage stability were $<4.92 \%$. The recovery was performed by adding known amount of individual standards into an accurately weighed sample, and the mixture was processed 
and analyzed by the same methods of the samples. The recoveries were in the range of $94.03 \%$ and $106.33 \%$ for the 41 compounds and the RSDs were $<3.76 \%$. Besides, no significant matrix effects were noted in relatively complex functional food matrices within $24 \mathrm{~h}$. All of above indicated that the established method was accurate enough for the determination of the 41 amino acids, nucleosides and nucleobases in S. nudus (Table 2). We have compared the determination results with and without internal standards in our preliminary experiments. The results showed that the optimized conditions of sample preparation, chromatogram and mass spectrum were stable and the contents of the components tested in the samples were quite identical, besides there was little difference between the errors of the methodology both with and without internal standards, so consequently, we decided not to use internal standards by reference to relevant methods in the field $[29,30]$.

\subsection{Sample Analysis}

The method was applied to analyze 25 free amino acids and 16 nucleosides and nucleobases in different parts of $S$. nudus collected from four different habitats in China with different sample preparation methods. The results demonstrated that all the samples were rich in these 41 compounds, and the total contents of these investigated compounds varied from $111.70 \mathrm{mg} / \mathrm{g}$ to $268.55 \mathrm{mg} / \mathrm{g}$. From the data, it was found that total contents of free amino acids, nucleosides and nucleobases in $S$. nudus extract processed by both ultrasonication and refluxing were pretty much the same or just a little decreased during by the refluxing procedure, the details of which are shown in Table 3. However, the total contents of three parts in S. nudus were different, and in the order: (highest) coelomic fluid > body wall > intestine (lowest). As for the habitats, the results showed that $S$. nudus collected from Hong Kong was low in nutritional value compared to the other habitats, while the qualities of the other three habitats are not too different from each other. From the perspective of shape and appearance, it was also proved that the worms inhabiting the waters surrounding Hong Kong are thinner, shorter, have a darker color and contain more silt (Figure 2).

For specific compounds determined in the experiments, remarkable differences were also observed. The contents of free amino acids except GABA were of milligram magnitude, while the contents of nucleosides or nucleobases were mostly of microgram magnitude. The contents of nucleosides or nucleobases were in the following order: nucleobases $>$ deoxynucleosides $>$ ribonucleosides. The concentrations of $2^{\prime}$-deoxyguanosine and its corresponding base were greater than any other nucleosides or nucleobases in all the parts.

2'-Deoxyguanosine is recognized as an oxidative damage biomarker of DNA [31]. The high contents of 2 -deoxyguanosine in the worms may be a result of physical and chemical factors such as foreign chemicals or ionizing radiation besides body metabolism. Additionally, the content of xanthine, reported to be bronchodilator, is of milligram magnitude and second to 2'-deoxyguanosine, which is extremely interesting and worthy of further study. As for free amino acids, S. nudus contains all 20 proteinogenic amino acids and five non-protein amino acids. The percentage of eight essential amino acids in the 20 proteinogenic amino acids varied from $23.91 \%$ to $33.11 \%$, and the percentage in body wall and intestine was higher than in coelomic fluid. The total contents of five non-protein amino acids in the samples, including GABA, citrulline, hydroxyproline, ornithine and taurine, varied from $7.77 \mathrm{mg} / \mathrm{g}$ to $22.90 \mathrm{mg} / \mathrm{g}$, among which the taurine had the highest percentage which varied from $84.60 \%$ to $94.60 \%$. Taurine has been reported to improve aerobic endurance, mental performance, concentration and memory [32], and we suggest that it may play an active role in the reported learning and memory enhancing pharmacological action as well as the anti-fatigue and anti-anoxia action of S. nudus [33,34]. Notably, the contents of fresh flavor amino acids including glycine, alanine, glutamate, aspartate and so on were fairly high, especially the contents of glycine which were the highest of all the free amino acids in S. nudus according to the assays, and all of these fresh flavor amino acids above fully explain its reputed good taste (Table 3). 
Table 2. Calibration curves, LOD, LOQ, precision, repeatability, stability and recovery of the 41 analytes.

\begin{tabular}{|c|c|c|c|c|c|c|c|c|c|c|c|c|}
\hline \multirow{2}{*}{ Analyte $^{a}$} & \multirow{2}{*}{ Calibration Curve } & \multirow{2}{*}{$r^{2}$} & \multirow{2}{*}{$\begin{array}{c}\text { Linear Range } \\
(\mu \mathrm{g} / \mathrm{mL})\end{array}$} & \multirow{2}{*}{$\begin{array}{c}\text { LOD } \\
(\mu \mathrm{g} / \mathrm{mL})\end{array}$} & \multirow{2}{*}{$\begin{array}{c}\mathrm{LOQ} \\
(\mu \mathrm{g} / \mathrm{mL})\end{array}$} & \multicolumn{2}{|c|}{ Precision RSD, $\%, n=6$} & \multirow{2}{*}{$\begin{array}{c}\text { Repeatablity } \\
(\text { RSD } \%)(n=6)\end{array}$} & \multirow{2}{*}{$\begin{array}{c}\text { Stability } \\
(\text { RSD } \%)(n=6)\end{array}$} & \multicolumn{2}{|c|}{ Recovery $(\%, n=3)$} & \multirow{2}{*}{$\begin{array}{l}\text { Matrix } \\
\text { Effect }^{\text {b }}\end{array}$} \\
\hline & & & & & & Intraday & Interday & & & Mean & RSD & \\
\hline 1 & $\mathrm{y}=3470.2 X-4115.1$ & 0.9975 & $0.57-28.25$ & 0.023 & 0.077 & 1.34 & 0.89 & 3.05 & 3.71 & 99.72 & 1.91 & 0.95 \\
\hline 2 & $y=17,200.4 X+4024.2$ & 0.9982 & $0.15-7.69$ & 0.021 & 0.069 & 1.02 & 1.03 & 3.03 & 4.92 & 99.86 & 2.87 & 0.92 \\
\hline 3 & $\mathrm{y}=12,693.4 \mathrm{X}+481.18$ & 0.9998 & $0.24-11.75$ & 0.007 & 0.025 & 0.75 & 0.39 & 2.57 & 2.27 & 105.13 & 3.47 & 1.02 \\
\hline 4 & $\mathrm{y}=5675.6 X-847.53$ & 0.9951 & $1.17-58.50$ & 0.033 & 0.110 & 2.84 & 2.92 & 3.12 & 4.90 & 98.65 & 1.11 & 0.93 \\
\hline 5 & $y=651.92 X+50.414$ & 0.9996 & $0.51-25.25$ & 0.080 & 0.267 & 2.65 & 2.90 & 2.38 & 4.66 & 94.64 & 1.54 & 0.98 \\
\hline 6 & $y=8691.2 X-303.63$ & 0.9999 & $1.09-54.50$ & 0.015 & 0.050 & 2.03 & 2.22 & 3.03 & 3.43 & 98.56 & 2.54 & 0.92 \\
\hline 7 & $\mathrm{y}=5984.3 \mathrm{X}-5771.5$ & 0.9997 & $0.61-30.25$ & 0.032 & 0.106 & 1.42 & 1.05 & 1.73 & 2.15 & 102.73 & 2.01 & 0.94 \\
\hline 8 & $y=13,359.8 x-12,482$ & 0.9992 & $0.49-24.50$ & 0.083 & 0.278 & 1.43 & 0.70 & 3.11 & 2.17 & 104.49 & 2.41 & 0.96 \\
\hline 9 & $\mathrm{y}=31,588 X-42,789$ & 0.9928 & $0.29-14.50$ & 0.003 & 0.008 & 2.98 & 3.02 & 2.55 & 1.36 & 99.59 & 3.14 & 1.01 \\
\hline 10 & $\mathrm{y}=11,200 \mathrm{x}-9094.5$ & 0.9961 & $0.86-43.13$ & 0.012 & 0.041 & 3.72 & 2.74 & 2.12 & 3.31 & 101.85 & 2.86 & 0.97 \\
\hline 11 & $y=10,862.6 x+510.08$ & 0.9999 & $1.60-79.69$ & 0.078 & 0.261 & 0.99 & 1.03 & 3.71 & 2.42 & 98.43 & 1.13 & 0.94 \\
\hline 12 & $y=83,008 X+23,366$ & 0.9923 & $0.11-5.37$ & 0.040 & 0.131 & 1.04 & 1.16 & 2.73 & 2.90 & 101.53 & 3.76 & 0.93 \\
\hline 13 & $\mathrm{y}=338.72 \mathrm{X}-22.948$ & 0.9985 & $0.29-14.25$ & 0.074 & 0.247 & 0.88 & 0.98 & 1.51 & 3.60 & 98.01 & 1.76 & 1.03 \\
\hline 14 & $\mathrm{y}=1246.64 X-83.519$ & 0.9999 & $0.56-27.75$ & 0.204 & 0.681 & 1.39 & 1.98 & 2.43 & 3.12 & 99.76 & 2.03 & 0.98 \\
\hline 15 & $y=3587.4 X+351.61$ & 0.9995 & $1.19-59.38$ & 0.021 & 0.071 & 3.10 & 3.42 & 2.76 & 3.39 & 100.04 & 2.86 & 0.91 \\
\hline 16 & $\mathrm{y}=396.14 X-47.85$ & 0.9969 & $0.35-17.66$ & 0.229 & 0.763 & 1.52 & 1.53 & 3.01 & 2.55 & 102.24 & 1.78 & 0.93 \\
\hline 17 & $y=457 X+281.7$ & 0.9973 & $1.80-90.00$ & 0.204 & 0.683 & 0.98 & 0.15 & 2.78 & 2.91 & 102.08 & 2.18 & 0.95 \\
\hline 18 & $y=66,192 X+262.14$ & 0.9919 & $0.29-14.44$ & 0.031 & 0.105 & 0.25 & 0.29 & 2.33 & 3.88 & 106.69 & 0.64 & 0.97 \\
\hline 19 & $\mathrm{y}=66,398 \mathrm{X}+140,788$ & 0.9925 & $1.78-89.07$ & 0.018 & 0.059 & 0.45 & 0.51 & 2.53 & 3.95 & 96.86 & 3.07 & 0.92 \\
\hline 20 & $\mathrm{y}=33,258 \mathrm{X}+18,095$ & 0.9922 & $0.31-15.23$ & 0.031 & 0.105 & 0.76 & 0.77 & 1.72 & 4.23 & 104.21 & 3.08 & 0.96 \\
\hline 21 & $y=11,852.6 x-1761.7$ & 0.9997 & $0.91-45.70$ & 0.016 & 0.055 & 1.14 & 0.93 & 2.70 & 3.11 & 101.27 & 2.36 & 0.97 \\
\hline 22 & $y=29,260 X+15,598$ & 0.9968 & $0.34-16.88$ & 0.023 & 0.076 & 1.93 & 2.13 & 2.13 & 2.10 & 99.63 & 1.53 & 0.92 \\
\hline 23 & $y=10,330.4 x+358.31$ & 0.9999 & $1.95-97.66$ & 0.040 & 0.132 & 0.57 & 0.58 & 1.36 & 4.33 & 100.78 & 3.09 & 0.93 \\
\hline 24 & $y=6520.8 X+1836.6$ & 0.9999 & $0.57-28.28$ & 0.060 & 0.200 & 1.39 & 1.37 & 1.44 & 3.86 & 99.45 & 2.60 & 0.93 \\
\hline 25 & $y=103.41 x+374.97$ & 0.9940 & $0.35-17.70$ & 0.098 & 0.325 & 1.75 & 2.01 & 3.33 & 3.31 & 104.37 & 1.47 & 1.02 \\
\hline 26 & $y=2565.1 x+1383.7$ & 0.9992 & $0.34-16.88$ & 0.037 & 0.121 & 0.95 & 1.00 & 1.19 & 2.94 & 95.94 & 2.51 & 0.99 \\
\hline 27 & $\mathrm{y}=8145.2 \mathrm{X}-3078$ & 0.9985 & $0.22-10.98$ & 0.023 & 0.075 & 0.60 & 0.56 & 3.29 & 2.77 & 100.14 & 3.17 & 0.92 \\
\hline 28 & $y=4690.1 x+1299.4$ & 0.9995 & $0.22-11.21$ & 0.029 & 0.096 & 0.55 & 0.62 & 2.93 & 3.64 & 97.65 & 2.92 & 0.94 \\
\hline 29 & $y=8301.8 x-1385.6$ & 0.9994 & $6.94-346.88$ & 0.060 & 0.202 & 0.84 & 0.91 & 1.98 & 4.85 & 94.03 & 3.33 & 0.96 \\
\hline 30 & $y=5115.8 x+1696$ & 0.9995 & $0.51-25.59$ & 0.028 & 0.092 & 0.86 & 0.67 & 0.88 & 2.67 & 106.07 & 2.09 & 1.01 \\
\hline 31 & $y=87,432 x+28,019$ & 0.9966 & $1.92-96.09$ & 0.031 & 0.105 & 1.45 & 1.48 & 2.35 & 3.05 & 96.84 & 3.93 & 0.95 \\
\hline 32 & $\mathrm{y}=2526.8 \mathrm{X}-1496.4$ & 0.9968 & $0.34-16.80$ & 0.026 & 0.086 & 0.99 & 1.07 & 1.03 & 2.58 & 98.26 & 2.16 & 0.95 \\
\hline 33 & $\mathrm{y}=1985.54 X-1440$ & 0.9999 & $0.07-3.42$ & 0.074 & 0.246 & 0.83 & 0.67 & 1.78 & 2.56 & 99.55 & 2.74 & 1.03 \\
\hline 34 & $y=5755.6 x-3405.1$ & 0.9994 & $1.64-82.03$ & 0.028 & 0.094 & 1.02 & 0.96 & 1.90 & 3.34 & 100.67 & 2.72 & 0.97 \\
\hline 35 & $y=1091.9 X+198.76$ & 0.9986 & $0.60-30.16$ & 0.048 & 0.161 & 1.07 & 1.23 & 2.21 & 3.36 & 103.23 & 1.74 & 0.92 \\
\hline 36 & $y=42,856 X-4351.9$ & 0.9999 & $1.82-91.02$ & 0.027 & 0.090 & 1.20 & 0.99 & 1.37 & 2.47 & 95.06 & 2.67 & 0.94 \\
\hline 37 & $\mathrm{y}=8726.2 \mathrm{X}-7910.9$ & 0.9986 & $0.89-44.53$ & 0.051 & 0.170 & 1.68 & 1.44 & 0.98 & 1.02 & 103.28 & 2.38 & 0.91 \\
\hline 38 & $y=3054.6 X+100.91$ & 0.9999 & $3.75-187.50$ & 0.106 & 0.352 & 1.45 & 1.68 & 1.90 & 3.04 & 105.55 & 1.85 & 0.98 \\
\hline 39 & $y=10,841.6 x-6708.3$ & 0.9996 & $7.81-390.63$ & 0.050 & 0.168 & 1.23 & 0.96 & 4.48 & 2.46 & 102.56 & 2.83 & 0.97 \\
\hline 40 & $y=12,365.8 X+2691.9$ & 0.9990 & $1.83-91.41$ & 0.018 & 0.060 & 1.51 & 0.55 & 3.15 & 2.88 & 100.41 & 3.07 & 0.95 \\
\hline 41 & $y=11,109.2 x+30,682$ & 0.9998 & $0.60-30.16$ & 0.024 & 0.080 & 2.08 & 1.42 & 2.57 & 1.35 & 106.33 & 1.50 & 0.91 \\
\hline
\end{tabular}

${ }^{\text {a }}$ Analyte number. ${ }^{\mathrm{b}}$ Matrix effects are calculated by slope matrix/slope solvent. 
Table 3. Contents (mg/g) of amino acids, nucleosides and nucleobases in Sipunculus nudus.

\begin{tabular}{|c|c|c|c|c|c|c|c|c|c|c|}
\hline \multirow{2}{*}{ Analyte ${ }^{a}$} & \multicolumn{10}{|c|}{ Samples $(\mathrm{mg} / \mathrm{g}, n=3)$} \\
\hline & H1 & G1 & S1 & F1 & H2 & G2 & S2 & F2 & G3 & S3 \\
\hline 1 & $0.15 \pm 0.02$ & $0.15 \pm 0.02$ & $0.18 \pm 0.02$ & $0.18 \pm 0.01$ & $0.18 \pm 0.03$ & $0.18 \pm 0.00$ & $0.21 \pm 0.01$ & $0.18 \pm 0.01$ & $0.27 \pm 0.03$ & $0.25 \pm 0.04$ \\
\hline 2 & $0.03 \pm 0.01$ & $0.01 \pm 0.00$ & $0.06 \pm 0.01$ & $0.04 \pm 0.01$ & $0.19 \pm 0.02$ & $0.10 \pm 0.02$ & $0.04 \pm 0.02$ & $0.04 \pm 0.00$ & $0.12 \pm 0.02$ & $0.05 \pm 0.01$ \\
\hline 3 & $0.14 \pm 0.04$ & $0.05 \pm 0.01$ & $0.09 \pm 0.01$ & $0.06 \pm 0.02$ & $0.08 \pm 0.01$ & $0.33 \pm 0.04$ & $0.13 \pm 0.01$ & $0.08 \pm 0.02$ & $0.21 \pm 0.02$ & $0.10 \pm 0.01$ \\
\hline 4 & $0.90 \pm 0.09$ & $1.30 \pm 0.28$ & $0.97 \pm 0.06$ & $1.78 \pm 1.21$ & $0.73 \pm 0.12$ & $0.63 \pm 0.09$ & $1.11 \pm 0.39$ & $0.98 \pm 0.05$ & $2.84 \pm 0.96$ & $3.22 \pm 0.24$ \\
\hline 5 & $0.16 \pm 0.01$ & $0.70 \pm 0.02$ & $0.38 \pm 0.03$ & $0.75 \pm 0.06$ & $0.16 \pm 0.02$ & $0.18 \pm 0.00$ & $0.45 \pm 0.04$ & $0.53 \pm 0.06$ & $1.22 \pm 0.22$ & $1.18 \pm 0.24$ \\
\hline 6 & $0.70 \pm 0.08$ & $1.24 \pm 0.21$ & $0.83 \pm 0.03$ & $1.80 \pm 0.51$ & $0.41 \pm 0.02$ & $0.44 \pm 0.03$ & $0.89 \pm 0.04$ & $0.64 \pm 0.02$ & $2.66 \pm 0.18$ & $2.86 \pm 0.16$ \\
\hline 7 & $0.16 \pm 0.01$ & $0.18 \pm 0.01$ & $0.19 \pm 0.02$ & $0.18 \pm 0.00$ & $0.16 \pm 0.02$ & $0.17 \pm 0.01$ & $0.19 \pm 0.01$ & $0.16 \pm 0.02$ & $0.31 \pm 0.02$ & $0.27 \pm 0.04$ \\
\hline 8 & $0.11 \pm 0.02$ & $0.15 \pm 0.01$ & $0.13 \pm 0.00$ & $0.16 \pm 0.02$ & $0.24 \pm 0.04$ & $0.17 \pm 0.01$ & $0.26 \pm 0.04$ & $0.27 \pm 0.04$ & $0.35 \pm 0.05$ & $0.36 \pm 0.06$ \\
\hline 9 & $0.55 \pm 0.17$ & $0.21 \pm 0.14$ & $0.41 \pm 0.08$ & $0.30 \pm 0.03$ & $0.43 \pm 0.01$ & $0.43 \pm 0.02$ & $0.28 \pm 0.03$ & $0.17 \pm 0.02$ & $0.34 \pm 0.04$ & $0.41 \pm 0.03$ \\
\hline 10 & $12.34 \pm 1.31$ & $21.45 \pm 0.68$ & $11.50 \pm 0.47$ & $25.07 \pm 1.68$ & $6.63 \pm 0.86$ & $7.57 \pm 0.61$ & $15.41 \pm 1.13$ & $11.61 \pm 0.42$ & $31.53 \pm 3.17$ & $28.66 \pm 2.16$ \\
\hline 11 & $1.19 \pm 0.31$ & $1.52 \pm 0.22$ & $1.24 \pm 0.41$ & $2.17 \pm 0.26$ & $0.94 \pm 0.16$ & $0.87 \pm 0.13$ & $1.33 \pm 0.06$ & $1.19 \pm 0.28$ & $3.15 \pm 0.04$ & $3.51 \pm 0.65$ \\
\hline 12 & $0.08+0.01$ & $0.18+0.01$ & $0.12+0.02$ & $0.14+0.01$ & $0.07+0.00$ & $0.06+0.01$ & $0.12+0.03$ & $0.14+0.00$ & $0.28+0.01$ & $0.22+0.02$ \\
\hline 13 & $0.10 \pm 0.02$ & $0.10 \pm 0.01$ & $0.12 \pm 0.01$ & $0.09 \pm 0.01$ & $0.07 \pm 0.01$ & $0.55 \pm 0.01$ & $0.85 \pm 0.12$ & $0.09 \pm 0.00$ & $0.07 \pm 0.01$ & $0.19 \pm 0.01$ \\
\hline 14 & $0.18+0.04$ & $0.62+0.08$ & $0.37+0.01$ & $0.62+0.03$ & $0.17+0.01$ & $0.11+0.00$ & $0.40+0.02$ & $0.37+0.03$ & $1.25+0.16$ & $1.40+0.03$ \\
\hline 15 & $1.60 \pm 0.34$ & $2.48 \pm 0.64$ & $1.85 \pm 0.67$ & $2.59 \pm 0.74$ & $1.27 \pm 0.17$ & $1.49 \pm 0.37$ & $1.92 \pm 0.34$ & $1.89 \pm 0.24$ & $4.08 \pm 0.07$ & $3.17 \pm 0.35$ \\
\hline 16 & $7.01 \pm 0.89$ & $11.39 \pm 1.68$ & $8.44 \pm 0.53$ & $11.36 \pm 1.23$ & $5.84 \pm 0.75$ & $6.60 \pm 0.61$ & $8.61 \pm 0.62$ & $8.39 \pm 0.32$ & $17.91 \pm 1.21$ & $14.36 \pm 0.81$ \\
\hline 17 & $26.84 \pm 2.14$ & $50.01 \pm 4.95$ & $30.62 \pm 2.87$ & $45.00 \pm 4.61$ & $18.07 \pm 1.14$ & $12.79 \pm 0.81$ & $25.86 \pm 2.27$ & $26.42 \pm 2.12$ & $60.59 \pm 4.65$ & $63.16 \pm 5.21$ \\
\hline 18 & $0.07 \pm 0.01$ & $0.06 \pm 0.00$ & $0.05 \pm 0.01$ & $0.05 \pm 0.02$ & $0.03 \pm 0.00$ & $0.03 \pm 0.01$ & $0.03 \pm 0.00$ & $0.05 \pm 0.01$ & $0.06 \pm 0.00$ & $0.05 \pm 0.02$ \\
\hline 19 & $5.03 \pm 0.87$ & $5.43 \pm 1.01$ & $4.95 \pm 0.21$ & $5.70 \pm 0.28$ & $2.64 \pm 0.75$ & $3.89 \pm 0.21$ & $3.68 \pm 0.35$ & $2.82 \pm 0.76$ & $5.21 \pm 1.01$ & $5.29 \pm 0.78$ \\
\hline 20 & $3.13 \pm 0.33$ & $4.27 \pm 0.36$ & $3.50 \pm 1.08$ & $4.19 \pm 0.51$ & $1.48 \pm 0.26$ & $2.57 \pm 0.13$ & $2.25 \pm 0.12$ & $1.86 \pm 0.73$ & $4.39 \pm 0.64$ & $4.55 \pm 0.17$ \\
\hline 21 & $0.85 \pm 0.02$ & $0.91 \pm 0.01$ & $0.81 \pm 0.02$ & $0.88 \pm 0.01$ & $1.36 \pm 0.48$ & $0.52 \pm 0.05$ & $0.48 \pm 0.09$ & $0.37 \pm 0.03$ & $0.76 \pm 0.01$ & $0.84 \pm 0.16$ \\
\hline 22 & $5.21 \pm 1.18$ & $6.19 \pm 1.22$ & $5.60 \pm 0.52$ & $6.47 \pm 0.79$ & $2.70 \pm 1.03$ & $4.39 \pm 0.23$ & $5.00 \pm 1.24$ & $3.59 \pm 0.12$ & $5.62 \pm 0.46$ & $5.81 \pm 0.74$ \\
\hline 23 & $0.93 \pm 0.03$ & $1.60 \pm 0.06$ & $1.14 \pm 0.03$ & $1.52 \pm 0.04$ & $0.55 \pm 0.05$ & $0.86 \pm 0.04$ & $1.09 \pm 0.02$ & $0.88 \pm 0.04$ & $1.65 \pm 0.12$ & $1.53 \pm 0.05$ \\
\hline 24 & $12.21 \pm 0.81$ & $13.06 \pm 1.12$ & $14.89 \pm 0.78$ & $12.03 \pm 1.12$ & $7.57 \pm 0.54$ & $9.03 \pm 0.48$ & $7.59 \pm 1.08$ & $6.32 \pm 0.19$ & $13.62 \pm 0.64$ & $13.54 \pm 0.61$ \\
\hline 25 & $4.31 \pm 0.19$ & $6.16 \pm 0.45$ & $4.71 \pm 0.73$ & $5.71 \pm 0.68$ & $2.91 \pm 0.14$ & $2.76 \pm 0.14$ & $3.15 \pm 0.19$ & $3.35 \pm 0.23$ & $5.91 \pm 0.37$ & $6.24 \pm 0.65$ \\
\hline 26 & $4.21 \pm 0.28$ & $5.98 \pm 0.26$ & $4.94 \pm 0.17$ & $5.25 \pm 0.42$ & $2.01 \pm 0.16$ & $2.68 \pm 0.17$ & $3.09 \pm 0.19$ & $2.27 \pm 0.31$ & $4.53 \pm 0.51$ & $5.37 \pm 1.01$ \\
\hline 27 & $3.31+0.34$ & $3.35+0.23$ & $3.11+0.07$ & $3.42+0.23$ & $1.75+0.45$ & $2.10+0.21$ & $2.49+1.81$ & $1.85+0.38$ & $3.72+0.19$ & $4.33+0.51$ \\
\hline 28 & $2.07 \pm 0.11$ & $2.25 \pm 0.04$ & $2.26 \pm 0.16$ & $2.71 \pm 0.12$ & $1.16 \pm 0.27$ & $2.12 \pm 0.13$ & $2.28 \pm 0.67$ & $1.53 \pm 0.05$ & $2.82 \pm 0.14$ & $3.36 \pm 0.31$ \\
\hline 29 & $8.54+0.57$ & $10.94+0.53$ & $8.90+1.01$ & $9.97+0.64$ & $5.18+0.45$ & $5.82+0.36$ & $6.54+0.38$ & $5.29+0.45$ & $11.43+0.91$ & $11.87+0.87$ \\
\hline 30 & $0.57 \pm 0.02$ & $0.48 \pm 0.04$ & $0.53 \pm 0.03$ & $0.49 \pm 0.02$ & $0.61 \pm 0.02$ & $0.61 \pm 0.03$ & $0.52 \pm 0.02$ & $0.72 \pm 0.03$ & $1.04 \pm 0.04$ & $0.78 \pm 0.08$ \\
\hline 31 & $2.44 \pm 0.27$ & $2.87 \pm 0.53$ & $2.87 \pm 0.32$ & $2.76 \pm 0.54$ & $1.57 \pm 0.21$ & $1.91 \pm 0.09$ & $1.64 \pm 0.12$ & $1.57 \pm 0.21$ & $3.49 \pm 0.11$ & $3.36 \pm 0.02$ \\
\hline 32 & $0.24 \pm 0.01$ & $0.25 \pm 0.02$ & $0.25 \pm 0.02$ & $0.27 \pm 0.01$ & $0.24 \pm 0.00$ & $0.24 \pm 0.01$ & $0.26 \pm 0.01$ & $0.23 \pm 0.00$ & $0.42 \pm 0.02$ & $0.43 \pm 0.02$ \\
\hline 33 & $0.09 \pm 0.00$ & $0.13 \pm 0.02$ & $0.10 \pm 0.01$ & $0.14 \pm 0.01$ & $0.11 \pm 0.02$ & $0.10 \pm 0.01$ & $0.10 \pm 0.01$ & $0.09 \pm 0.00$ & $0.17 \pm 0.01$ & $0.17 \pm 0.01$ \\
\hline 34 & $4.03 \pm 0.09$ & $4.71 \pm 0.15$ & $4.26 \pm 0.13$ & $4.62 \pm 0.07$ & $1.73 \pm 0.37$ & $2.32 \pm 1.56$ & $2.98 \pm 1.34$ & $1.94 \pm 0.21$ & $3.68 \pm 1.11$ & $3.83 \pm 0.21$ \\
\hline 35 & $21.43 \pm 2.01$ & $16.09 \pm 1.97$ & $15.59 \pm 1.12$ & $14.89 \pm 0.21$ & $15.80 \pm 0.21$ & $8.29 \pm 1.18$ & $9.36 \pm 0.45$ & $8.81 \pm 1.17$ & $19.61 \pm 1.24$ & $20.77 \pm 1.58$ \\
\hline
\end{tabular}


Table 3. Cont.

\begin{tabular}{|c|c|c|c|c|c|c|c|c|c|c|}
\hline \multirow{2}{*}{ Analyte ${ }^{a}$} & \multicolumn{10}{|c|}{ Samples $(\mathrm{mg} / \mathrm{g}, n=3)$} \\
\hline & H1 & G1 & S1 & F1 & H2 & G2 & S2 & F2 & G3 & S3 \\
\hline 36 & $3.83 \pm 1.12$ & $4.59 \pm 1.57$ & $3.73 \pm 1.16$ & $4.29 \pm 1.66$ & $2.16 \pm 0.57$ & $2.62 \pm 0.07$ & $2.48 \pm 0.08$ & $2.15 \pm 0.58$ & $4.77 \pm 1.56$ & $4.82 \pm 1.45$ \\
\hline 37 & $0.43 \pm 0.02$ & $0.52 \pm 0.02$ & $0.50 \pm 0.01$ & $0.46 \pm 0.00$ & $0.45 \pm 0.01$ & $0.61 \pm 0.03$ & $0.39 \pm 0.02$ & $0.39 \pm 0.03$ & $1.19 \pm 0.21$ & $0.87 \pm 0.04$ \\
\hline 38 & $5.79 \pm 0.75$ & $8.95 \pm 0.58$ & $8.08 \pm 1.01$ & $8.87 \pm 1.22$ & $4.16 \pm 0.76$ & $5.48 \pm 1.43$ & $6.17 \pm 0.17$ & $4.22 \pm 0.13$ & $7.73 \pm 0.97$ & $8.52 \pm 1.04$ \\
\hline 39 & $11.85 \pm 0.74$ & $14.11 \pm 1.02$ & $12.64 \pm 0.35$ & $14.51 \pm 1.02$ & $7.86 \pm 0.13$ & $9.50 \pm 0.15$ & $8.75 \pm 1.62$ & $7.39 \pm 1.44$ & $15.07 \pm 1.21$ & $15.72 \pm 1.43$ \\
\hline 40 & $2.21 \pm 0.13$ & $2.81 \pm 0.14$ & $2.85 \pm 0.53$ & $2.98 \pm 0.18$ & $1.40 \pm 0.19$ & $1.76 \pm 0.48$ & $2.18 \pm 0.17$ & $1.68 \pm 0.15$ & $2.98 \pm 0.17$ & $3.00 \pm 0.81$ \\
\hline 41 & $14.70 \pm 0.47$ & $17.12 \pm 1.02$ & $13.96 \pm 1.02$ & $17.05 \pm 0.98$ & $12.47 \pm 2.01$ & $8.82 \pm 1.78$ & $12.41 \pm 0.45$ & $11.25 \pm 0.56$ & $18.76 \pm 1.87$ & $20.12 \pm 2.12$ \\
\hline $\mathrm{N}^{\mathrm{c}}$ & $25.40 \pm 3.81$ & $41.74 \pm 5.42$ & $26.89 \pm 2.83$ & $47.29 \pm 6.14$ & $17.59 \pm 2.15$ & $19.88 \pm 1.98$ & $32.20 \pm 2.21$ & $26.71 \pm 2.72$ & $66.57 \pm 4.72$ & $60.22 \pm 5.29$ \\
\hline$A^{d}$ & $144.32 \pm 15.61$ & $182.85 \pm 18.39$ & $150.86 \pm 12.70$ & $174.24 \pm 21.74$ & $95.97 \pm 8.45$ & $91.82 \pm 10.83$ & $110.78 \pm 14.92$ & $98.16 \pm 10.84$ & $199.25 \pm 17.81$ & $208.34 \pm 20.37$ \\
\hline Total & $169.71 \pm 15.78$ & $224.59 \pm 22.74$ & $177.74 \pm 16.82$ & $211.53 \pm 25.62$ & $113.56 \pm 13.00$ & $111.70 \pm 7.38$ & $142.98 \pm 14.71$ & $124.88 \pm 13.80$ & $265.81 \pm 22.89$ & $268.55 \pm 27.85$ \\
\hline \multirow{2}{*}{ Analyte $^{a}$} & \multicolumn{10}{|c|}{ Sample $(\mathrm{mg} / \mathrm{g}, n=3)$} \\
\hline & F3 & H4 & G4 & S4 & F4 & H5 & G5 & S5 & F5 & \\
\hline 1 & $0.27 \pm 0.03$ & $0.16 \pm 0.02$ & $0.15 \pm 0.02$ & $0.20 \pm 0.01$ & $0.18 \pm 0.00$ & $0.16 \pm 0.01$ & $0.15 \pm 0.02$ & $0.20 \pm 0.02$ & $0.17 \pm 0.01$ & \\
\hline 2 & $0.07 \pm 0.01$ & $0.16 \pm 0.02$ & $0.08 \pm 0.02$ & $0.09 \pm 0.01$ & $0.10 \pm 0.01$ & $0.25 \pm 0.02$ & $0.13 \pm 0.01$ & $0.05 \pm 0.00$ & $0.06 \pm 0.01$ & \\
\hline 3 & $0.12 \pm 0.03$ & $0.15 \pm 0.01$ & $0.10 \pm 0.01$ & $0.09 \pm 0.02$ & $0.08 \pm 0.02$ & $0.10 \pm 0.00$ & $0.33 \pm 0.02$ & $0.16 \pm 0.02$ & $0.09 \pm 0.02$ & \\
\hline 4 & $2.15 \pm 1.02$ & $0.88 \pm 0.05$ & $1.36 \pm 0.20$ & $0.89 \pm 0.08$ & $1.60 \pm 0.20$ & $0.60 \pm 0.05$ & $0.66 \pm 0.04$ & $0.92 \pm 0.05$ & $0.85 \pm 0.04$ & \\
\hline 5 & $1.20 \pm 0.38$ & $0.18 \pm 0.03$ & $0.61 \pm 0.04$ & $0.35 \pm 0.02$ & $0.57 \pm 0.02$ & $0.11 \pm 0.01$ & $0.15 \pm 0.05$ & $0.30 \pm 0.04$ & $0.44 \pm 0.03$ & \\
\hline 6 & $1.92 \pm 0.21$ & $0.69 \pm 0.23$ & $1.13 \pm 0.16$ & $0.74 \pm 0.02$ & $1.39 \pm 0.19$ & $0.40 \pm 0.03$ & $0.46 \pm 0.11$ & $0.68 \pm 0.04$ & $0.53 \pm 0.05$ & \\
\hline 7 & $0.26 \pm 0.05$ & $0.17 \pm 0.04$ & $0.18 \pm 0.03$ & $0.18 \pm 0.02$ & $0.17 \pm 0.06$ & $0.17 \pm 0.03$ & $0.17 \pm 0.03$ & $0.19 \pm 0.04$ & $0.18 \pm 0.06$ & \\
\hline 8 & $0.22 \pm 0.04$ & $0.12 \pm 0.03$ & $0.12 \pm 0.02$ & $0.12 \pm 0.01$ & $0.12 \pm 0.00$ & $0.12 \pm 0.01$ & $0.17 \pm 0.03$ & $0.17 \pm 0.04$ & $0.16 \pm 0.02$ & \\
\hline 9 & $0.73 \pm 0.05$ & $0.22 \pm 0.04$ & $0.22 \pm 0.03$ & $0.38 \pm 0.07$ & $0.35 \pm 0.08$ & $0.39 \pm 0.09$ & $0.44 \pm 0.03$ & $0.28 \pm 0.07$ & $0.18 \pm 0.02$ & \\
\hline 10 & $22.54 \pm 0.48$ & $11.81 \pm 0.38$ & $20.81 \pm 0.24$ & $11.23 \pm 0.45$ & $24.70 \pm 2.12$ & $5.91 \pm 0.17$ & $8.06 \pm 1.12$ & $11.81 \pm 1.32$ & $10.39 \pm 1.89$ & \\
\hline 11 & $2.46 \pm 0.34$ & $1.19 \pm 0.03$ & $1.76 \pm 0.12$ & $1.16 \pm 0.03$ & $2.23 \pm 0.34$ & $0.85 \pm 0.02$ & $0.81 \pm 0.03$ & $1.21 \pm 0.05$ & $1.22 \pm 0.04$ & \\
\hline 12 & $0.19 \pm 0.01$ & $0.07 \pm 0.00$ & $0.17 \pm 0.02$ & $0.10 \pm 0.01$ & $0.11 \pm 0.01$ & $0.07 \pm 0.01$ & $0.07 \pm 0.02$ & $0.09 \pm 0.03$ & $0.13 \pm 0.00$ & \\
\hline 13 & $0.21 \pm 0.04$ & $0.12 \pm 0.01$ & $0.09 \pm 0.02$ & $0.12 \pm 0.01$ & $0.10 \pm 0.01$ & $0.05 \pm 0.00$ & $0.52 \pm 0.01$ & $0.84 \pm 0.11$ & $0.07 \pm 0.02$ & \\
\hline 14 & $1.23 \pm 0.22$ & $0.15 \pm 0.01$ & $0.49 \pm 0.02$ & $0.36 \pm 0.03$ & $0.12 \pm 0.01$ & $0.10 \pm 0.01$ & $0.09 \pm 0.02$ & $0.26 \pm 0.05$ & $0.18 \pm 0.02$ & \\
\hline 15 & $2.70 \pm 0.28$ & $1.57 \pm 0.51$ & $2.55 \pm 0.35$ & $1.72 \pm 0.12$ & $2.41 \pm 0.33$ & $1.10 \pm 0.03$ & $1.44 \pm 0.21$ & $1.68 \pm 0.13$ & $1.76 \pm 0.22$ & \\
\hline 16 & $14.41 \pm 1.13$ & $7.17 \pm 1.07$ & $10.97 \pm 1.45$ & $7.90 \pm 0.65$ & $10.61 \pm 2.24$ & $5.06 \pm 0.07$ & $6.67 \pm 1.01$ & $7.39 \pm 0.23$ & $7.85 \pm 0.25$ & \\
\hline 17 & $61.03 \pm 3.43$ & $23.57 \pm 1.75$ & $44.71 \pm 2.69$ & $26.68 \pm 2.01$ & $32.35 \pm 2.84$ & $20.65 \pm 1.05$ & $15.25 \pm 0.98$ & $16.13 \pm 1.29$ & $21.27 \pm 2.13$ & \\
\hline 18 & $0.03 \pm 0.00$ & $0.04 \pm 0.01$ & $0.07 \pm 0.00$ & $0.04 \pm 0.01$ & $0.03 \pm 0.01$ & $0.03 \pm 0.01$ & $0.04 \pm 0.01$ & $0.03 \pm 0.01$ & $0.04 \pm 0.00$ & \\
\hline 19 & $4.37 \pm 0.19$ & $6.19 \pm 0.92$ & $6.25 \pm 0.83$ & $5.24 \pm 0.25$ & $5.56 \pm 0.24$ & $2.97 \pm 0.17$ & $3.89 \pm 0.35$ & $3.82 \pm 0.23$ & $3.05 \pm 1.62$ & \\
\hline 20 & $4.45 \pm 0.83$ & $3.51 \pm 0.57$ & $5.06 \pm 0.87$ & $3.53 \pm 1.17$ & $4.02 \pm 0.48$ & $1.39 \pm 0.49$ & $2.52 \pm 0.57$ & $2.23 \pm 0.18$ & $1.91 \pm 0.52$ & \\
\hline
\end{tabular}


Table 3. Cont

\begin{tabular}{|c|c|c|c|c|c|c|c|c|c|}
\hline \multirow{2}{*}{ Analyte $^{a}$} & \multicolumn{9}{|c|}{ Sample $(\mathrm{mg} / \mathrm{g}, n=3)$} \\
\hline & F3 & H4 & G4 & S4 & F4 & H5 & G5 & S5 & F5 \\
\hline 21 & $0.58 \pm 0.02$ & $0.91 \pm 0.11$ & $0.99 \pm 0.13$ & $0.84 \pm 0.03$ & $0.84 \pm 0.04$ & $1.36 \pm 0.26$ & $0.50 \pm 0.01$ & $1.44 \pm 0.22$ & $1.37 \pm 0.26$ \\
\hline 22 & $5.57 \pm 1.72$ & $6.16 \pm 1.52$ & $8.06 \pm 0.15$ & $5.85 \pm 0.18$ & $6.83 \pm 0.56$ & $2.89 \pm 0.82$ & $4.29 \pm 1.74$ & $5.48 \pm 0.32$ & $4.17 \pm 0.52$ \\
\hline 23 & $1.51 \pm 0.24$ & $1.10 \pm 0.04$ & $1.97 \pm 0.06$ & $1.18 \pm 0.05$ & $1.44 \pm 0.02$ & $0.54 \pm 0.03$ & $0.81 \pm 0.03$ & $1.07 \pm 0.04$ & $0.95 \pm 0.04$ \\
\hline 24 & $13.72 \pm 1.80$ & $11.77 \pm 0.59$ & $13.28 \pm 1.60$ & $13.36 \pm 1.80$ & $10.20 \pm 0.54$ & $7.73 \pm 0.65$ & $9.76 \pm 1.17$ & $6.46 \pm 0.54$ & $5.98 \pm 0.67$ \\
\hline 25 & $5.54 \pm 1.10$ & $4.55 \pm 1.04$ & $5.75 \pm 1.05$ & $4.01 \pm 0.24$ & $4.14 \pm 0.21$ & $2.49 \pm 0.14$ & $2.82 \pm 0.06$ & $2.71 \pm 0.58$ & $3.07 \pm 0.12$ \\
\hline 26 & $4.40 \pm 0.24$ & $4.22 \pm 0.36$ & $6.13 \pm 0.26$ & $4.83 \pm 0.19$ & $4.03 \pm 0.26$ & $2.05 \pm 0.17$ & $2.66 \pm 0.76$ & $2.72 \pm 0.86$ & $1.99 \pm 0.55$ \\
\hline 27 & $2.71 \pm 0.55$ & $3.17 \pm 0.15$ & $3.43 \pm 1.12$ & $2.97 \pm 0.32$ & $2.81 \pm 0.30$ & $2.11 \pm 1.01$ & $2.19 \pm 0.96$ & $2.12 \pm 0.18$ & $1.60 \pm 0.47$ \\
\hline 28 & $2.16 \pm 0.58$ & $1.66 \pm 0.24$ & $1.83 \pm 0.57$ & $1.92 \pm 0.57$ & $1.69 \pm 0.61$ & $1.12 \pm 0.17$ & $1.79 \pm 0.52$ & $1.89 \pm 0.57$ & $1.28 \pm 0.24$ \\
\hline 29 & $10.86 \pm 0.57$ & $8.19 \pm 0.35$ & $10.75 \pm 0.57$ & $8.20 \pm 0.43$ & $7.43 \pm 0.43$ & $4.92 \pm 0.81$ & $5.89 \pm 1.13$ & $5.31 \pm 0.52$ & $4.75 \pm 0.86$ \\
\hline 30 & $0.78 \pm 0.23$ & $0.47 \pm 0.02$ & $0.48 \pm 0.13$ & $0.52 \pm 0.21$ & $0.47 \pm 0.01$ & $0.52 \pm 0.22$ & $0.56 \pm 0.05$ & $0.53 \pm 0.04$ & $0.67 \pm 0.02$ \\
\hline 31 & $2.74 \pm 0.37$ & $2.36 \pm 0.48$ & $2.82 \pm 0.39$ & $2.67 \pm 0.37$ & $2.38 \pm 0.66$ & $1.55 \pm 0.21$ & $2.03 \pm 0.54$ & $1.39 \pm 0.23$ & $1.43 \pm 0.21$ \\
\hline 32 & $0.39 \pm 0.04$ & $0.24 \pm 0.01$ & $0.26 \pm 0.00$ & $0.25 \pm 0.02$ & $0.27 \pm 0.04$ & $0.25 \pm 0.02$ & $0.24 \pm 0.02$ & $0.25 \pm 0.03$ & $0.25 \pm 0.01$ \\
\hline 33 & $0.15 \pm 0.01$ & $+b$ & + & + & + & + & + & + & + \\
\hline 34 & $3.76 \pm 0.32$ & $4.50 \pm 0.74$ & $5.59 \pm 1.02$ & $4.41 \pm 1.02$ & $4.40 \pm 1.12$ & $1.79 \pm 0.47$ & $2.43 \pm 0.32$ & $3.02 \pm 0.16$ & $2.17 \pm 0.38$ \\
\hline 35 & $17.84 \pm 1.01$ & $20.10 \pm 1.29$ & $14.46 \pm 0.92$ & $13.68 \pm 0.62$ & $11.36 \pm 0.73$ & $17.36 \pm 1.21$ & $9.56 \pm 1.02$ & $6.62 \pm 0.24$ & $7.42 \pm 1.01$ \\
\hline 36 & $4.11 \pm 0.76$ & $4.32 \pm 0.58$ & $5.53 \pm 0.56$ & $3.84 \pm 1.03$ & $4.37 \pm 0.63$ & $2.17 \pm 0.98$ & $2.61 \pm 0.72$ & $2.56 \pm 0.36$ & $2.34 \pm 0.46$ \\
\hline 37 & $0.61 \pm 0.02$ & $0.43 \pm 0.01$ & $0.53 \pm 0.01$ & $0.44 \pm 0.00$ & $0.41 \pm 0.02$ & $0.47 \pm 0.01$ & $0.44 \pm 0.02$ & $0.34 \pm 0.00$ & $0.39 \pm 0.03$ \\
\hline 38 & $6.97 \pm 0.26$ & $5.22 \pm 0.83$ & $9.18 \pm 1.01$ & $7.81 \pm 0.41$ & $6.27 \pm 0.78$ & $3.05 \pm 0.15$ & $5.52 \pm 0.83$ & $5.12 \pm 0.45$ & $3.85 \pm 1.01$ \\
\hline 39 & $15.45 \pm 1.32$ & $16.40 \pm 1.28$ & $16.26 \pm 1.37$ & $13.70 \pm 0.82$ & $12.76 \pm 1.51$ & $8.55 \pm 0.72$ & $9.90 \pm 0.50$ & $8.02 \pm 0.23$ & $7.79 \pm 0.17$ \\
\hline 40 & $2.84 \pm 0.22$ & $2.23 \pm 0.67$ & $2.85 \pm 0.08$ & $2.61 \pm 0.45$ & $2.27 \pm 0.98$ & $1.28 \pm 0.16$ & $1.77 \pm 0.53$ & $1.89 \pm 0.34$ & $15.20 \pm 1.28$ \\
\hline 41 & $20.99 \pm 0.46$ & $15.13 \pm 0.98$ & $15.93 \pm 1.39$ & $12.30 \pm 0.78$ & $12.64 \pm 1.01$ & $12.05 \pm 0.94$ & $9.36 \pm 0.27$ & $9.16 \pm 0.43$ & $10.04 \pm 0.17$ \\
\hline $\mathrm{N}^{\mathrm{c}}$ & $50.69 \pm 4.81$ & $24.80 \pm 1.26$ & $40.80 \pm 4.19$ & $25.64 \pm 2.11$ & $44.85 \pm 3.62$ & $15.43 \pm 1.12$ & $20.33 \pm 2.03$ & $26.25 \pm 1.03$ & $24.35 \pm 0.78$ \\
\hline$A^{d}$ & $193.56 \pm 18.72$ & $146.48 \pm 15.29$ & $182.18 \pm 16.50$ & $140.91 \pm 14.93$ & $139.18 \pm 11.27$ & $99.83 \pm 10.83$ & $96.83 \pm 8.23$ & $90.35 \pm 12.47$ & $89.29 \pm 11.38$ \\
\hline Total & $244.25 \pm 22.51$ & $171.27 \pm 16.78$ & $222.98 \pm 23.85$ & $166.54 \pm 15.73$ & $184.03 \pm 18.35$ & $114.76 \pm 12.46$ & $117.16 \pm 15.47$ & $116.59 \pm 14.19$ & $113.64 \pm 13.86$ \\
\hline
\end{tabular}

The data was 


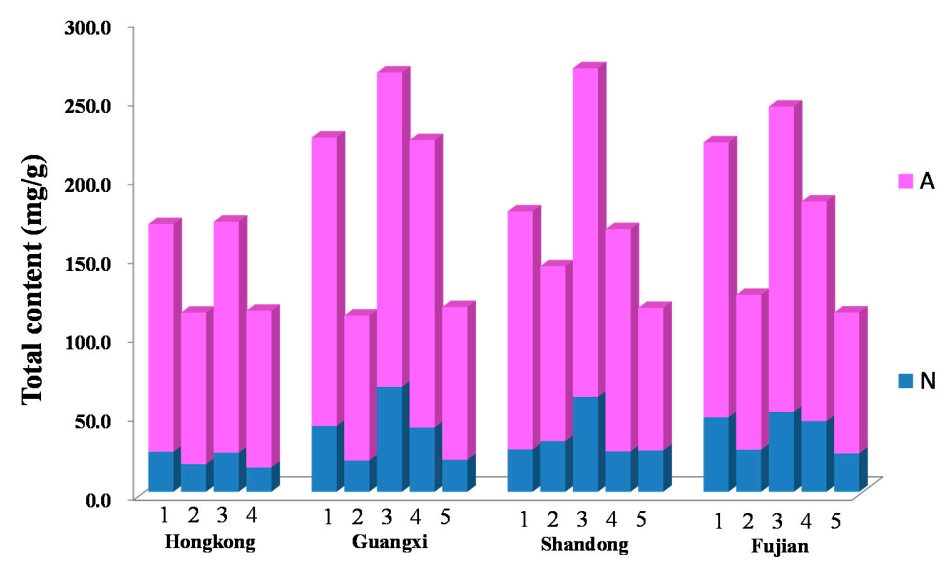

Figure 2. The total contents of 41 compounds in different samples. The numbers on the stacked bar chart indicate body wall, ultrasonication (1), intestine, ultrasonication (2), coelomic fluid, ultrasonication (3), body wall, refluxing (4) and intestine, refluxing (5). The color A stands for total contents of amino acids, and the color $\mathrm{N}$ stands for total contents of nucleosides and nucleobases.

To evaluate the variation of samples, PCA was performed on the basis of the contents of 41 tested compounds from UPLC-TQ-MS profiles. The first three principal components (PC 1, PC 2 and PC 3) with $>83.70 \%$ of the whole variance were extracted. Among them, PC 1, PC 2 and PC 3 accounted for $60.63,17.16$ and $5.91 \%$ of total variance, respectively. The remaining principal components, which had a minor effect on the model, were discarded. According to their loadings, PC 1 had good correlations with $2^{\prime}$-deoxyinosine, $2^{\prime}$-deoxyguanosine, guanine, xanthine, glycine, isoleucine, threonine, glutamate, proline and histidine, among which the correlation of glycine, threonine and glutamate are over $95 \%$. And PC 2 had good correlation with analytes of 2'-deoxyadenosine- $5^{\prime}$-monophosphate and citrulline whereas PC 3 had good correlation with analytes of inosine and taurine (Table 4). In the scatter plot, each sample is represented as a marker. It was noticeable that the samples were clearly clustered into three groups: cluster A (sample H2, F2, G2, S2, H5, F5, G5, S5), cluster B (H1, F1, G1, S1, H4, F4, G4, S4) and cluster C (F3, G3, S3) (Figure 3). This result indicated there are significant differences in the proportions and quantity of the 41 compounds in different parts in S. nudus while the impact of the extraction procedure method used was not significant.

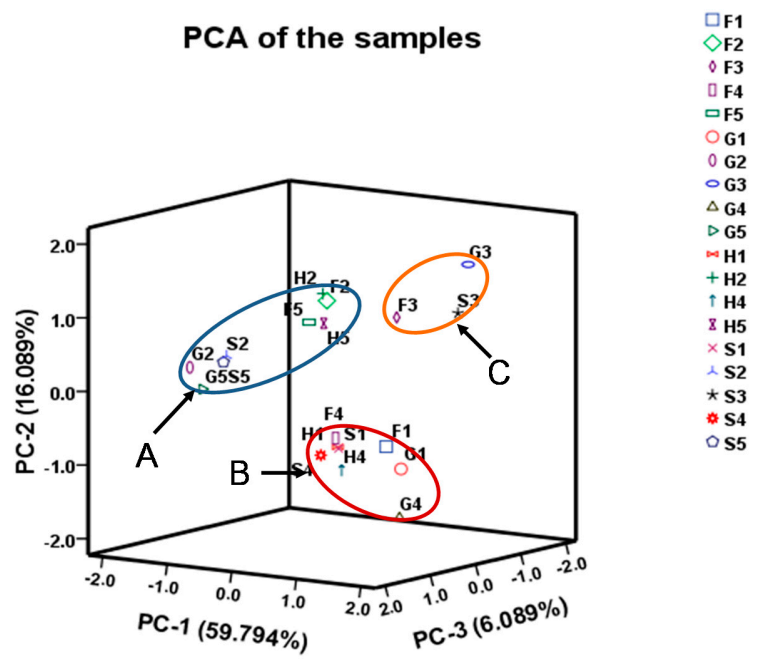

Figure 3. The PCA of the samples. The samples were body wall, ultrasonication (1), intestine, ultrasonication (2), coelomic fluid, ultrasonication (3), body wal, refluxing (4) and intestine refluxing (5) collected from Fujian (F), Guangxi (G), Hong Kong (H) and Shandong (S). 
Table 4. Component loading matrix of 41 amino acids, nucleosides and nucleobases for PCA.

\begin{tabular}{|c|c|c|c|c|c|c|c|c|c|c|c|c|c|c|c|c|c|c|c|c|c|}
\hline \multirow{2}{*}{$\begin{array}{l}\text { Component } \\
\text { (PC) }\end{array}$} & \multicolumn{21}{|c|}{ Analytes } \\
\hline & 1 & 2 & 3 & 4 & 5 & 6 & 7 & 8 & 9 & 10 & 11 & 12 & 13 & 14 & 15 & 16 & 17 & 18 & 19 & 20 & 21 \\
\hline 1 & 0.570 & -0.374 & -0.211 & 0.894 & 0.873 & 0.923 & 0.759 & 0.395 & 0.150 & 0.918 & 0.891 & 0.855 & -0.313 & 0.861 & 0.914 & 0.921 & 0.950 & 0.540 & 0.721 & 0.915 & 0.719 \\
\hline 2 & 0.695 & 0.146 & 0.236 & 0.376 & 0.384 & 0.310 & 0.574 & 0.813 & 0.242 & 0.106 & 0.319 & 0.347 & 0.215 & 0.446 & 0.257 & 0.296 & 0.156 & -0.364 & -0.624 & -0.330 & -0.660 \\
\hline \multirow[t]{2}{*}{3} & -0.053 & 0.667 & -0.017 & -0.041 & -0.150 & -0.044 & 0.039 & -0.121 & 0.494 & -0.202 & -0.059 & -0.146 & -0.605 & -0.015 & -0.173 & -0.127 & 0.036 & 0.130 & -0.003 & -0.049 & 0.061 \\
\hline & 22 & 23 & 24 & 25 & 26 & 27 & 28 & 29 & 30 & 31 & 32 & 33 & 34 & 35 & 36 & 37 & 38 & 39 & 40 & 41 & \\
\hline 1 & 0.688 & 0.872 & 0.811 & 0.954 & 0.842 & 0.891 & 0.808 & 0.969 & 0.426 & 0.935 & 0.787 & 0.630 & 0.708 & 0.595 & 0.884 & 0.715 & 0.836 & 0.858 & 0.930 & 0.899 & \\
\hline 2 & -0.578 & -0.233 & -0.357 & -0.199 & -0.509 & -0.273 & 0.142 & -0.170 & 0.800 & -0.173 & 0.556 & 0.448 & -0.677 & -0.106 & -0.417 & 0.479 & -0.357 & -0.374 & -0.241 & 0.051 & \\
\hline 3 & -0.324 & -0.328 & 0.296 & 0.048 & -0.031 & 0.105 & -0.221 & 0.084 & 0.136 & 0.185 & 0.110 & 0.049 & -0.094 & 0.724 & 0.057 & 0.215 & -0.210 & 0.196 & -0.115 & 0.266 & \\
\hline
\end{tabular}




\section{Materials and Methods}

\subsection{Reagents and Materials}

Ammonium formate (Shanghai Lingfeng Chemical Reagent Co., Ltd., Shanghai, China), ammonium acetate (Sinopharm Chemical Reagent Co., Ltd., Shanghai, China), formic acid (Merck Millipore, Darmstadt, Germany) and acetonitrile (Tedia China, Shanghai, China) were analytical grade. Deionized water $\left(\mathrm{H}_{2} \mathrm{O}\right)$ was purified by a superpurification system (EPED Technology Development, Nanjing, China). Forty one standards including adenosine- $5^{\prime}$-mono-phosphate (1), inosine (2), guanosine (3), thymidine (4), 2' -deoxyuridine (5), $2^{\prime}$-deoxyinosine (6), cytidine-5' ${ }^{\prime}$-monophosphate (7), $2^{\prime}$-deoxyadenosine- $5^{\prime}$-monophosphate (8), 2'-deoxycytidine (9), 2'-deoxyguanosine (10), thymine (11), adenine (12), cytidine (13), uracil (14), guanine (15), glycine (17), GABA (18), asparagine (27), glutamine (28), citrulline (30), hydroxyproline (32), taurine (35) and ornithine (37) were purchased from Sigma (St. Louis, MO, USA). Standards including xanthine (16), leucine (19), isoleucine (20), phenylalanine (22), tryptophan (23), alanine (24), threonine (25), serine (26), glutamate (29), proline (31), tyrosine (34), valine (36), aspartate (38), lysine (39), histidine (40) and arginine (41) were obtained from the National Institute for the Control of Pharmaceutical and Biological Products (Beijing, China). A chemical standard of cysteine (33) was obtained from Aladdin Chemical (Shanghai, China). A reference of methionine (21) was purchased from Sinopharm Chemical Reagent (Beijing, China). The purity of each compound was $>98 \%$, as determined by UPLC analysis (Figure 4).

A mixed standard stock solution containing the reference compounds 1-41 dried to constant weight was prepared in methanol/water $(9: 1, v / v)$. Working standard solutions for calibration curves were prepared by diluting the mixed standard stock solution with $10 \%$ methanol at different concentrations.

\subsection{Sample Preparation}

The samples included three different parts (body wall, intestine, coelomic fluid) of S. nudus collected from four different habitats in China in August, 2014 (Table 5).

Table 5. Sipunculus nudus samples.

\begin{tabular}{|c|c|c|c|c|}
\hline NO & Sample & Anatomical Part & Habitat & $\begin{array}{c}\text { Processing } \\
\text { Method }\end{array}$ \\
\hline 1 & $\mathrm{H} 1$ & Body wall (SE) & Hongkong & Ultrasonication \\
\hline 2 & G1 & Body wall (SE) & Guangxi & Ultrasonication \\
\hline 3 & S1 & Body wall (SE) & Shandong & Ultrasonication \\
\hline 4 & $\mathrm{~F} 1$ & Body wall (SE) & Fujian & Ultrasonication \\
\hline 5 & $\mathrm{H} 2$ & Intestine (SI) & Hongkong & Ultrasonication \\
\hline 6 & G2 & Intestine (SI) & Guangxi & Ultrasonication \\
\hline 7 & $\mathrm{~S} 2$ & Intestine (SI) & Shandong & Ultrasonication \\
\hline 8 & F2 & Intestine (SI) & Fujian & Ultrasonication \\
\hline 9 & G3 & Coelomic fluid (SC) & Guangxi & Ultrasonication \\
\hline 10 & S3 & Coelomic fluid (SC) & Shandong & Ultrasonication \\
\hline 11 & F3 & Coelomic fluid (SC) & Fujian & Ultrasonication \\
\hline 12 & $\mathrm{H} 4$ & Body wall (SE) & Hongkong & Refluxing \\
\hline 13 & G4 & Body wall (SE) & Guangxi & Refluxing \\
\hline 14 & $\mathrm{~S} 4$ & Body wall (SE) & Shandong & Refluxing \\
\hline 15 & $\mathrm{~F} 4$ & Body wall (SE) & Fujian & Refluxing \\
\hline 16 & H5 & Intestine (SI) & Hongkong & Refluxing \\
\hline 17 & G5 & Intestine (SI) & Guangxi & Refluxing \\
\hline 18 & S5 & Intestine (SI) & Shandong & Refluxing \\
\hline 19 & F5 & Intestine (SI) & Fujian & Refluxing \\
\hline
\end{tabular}

Three batches of each sample were collected from the different locations. 


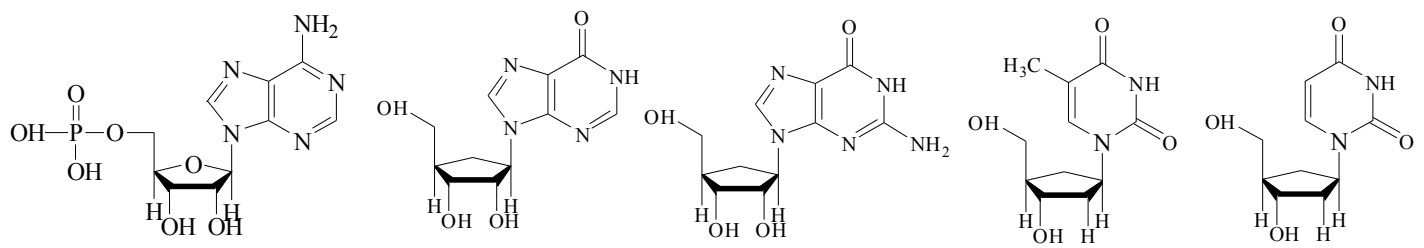

(1)Adenosine-5'-monophosphate (2) Inosine

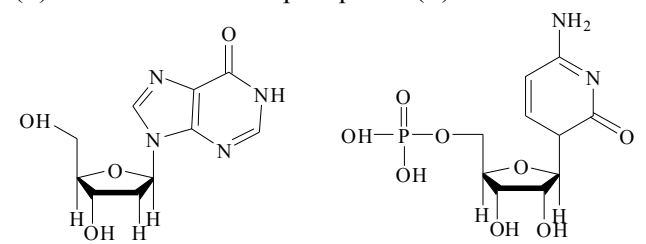

(3) Guanosine

(4) Thymidine

(5) 2'-deoxyuridine
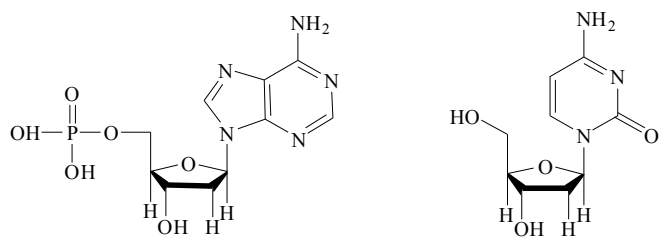

(6)2'-deoxyinosine (7)Cytidine-5'-monophosphate (8)2'-deoxycytidine-5'-monophosphate (9)2'-deoxycytidine<smiles>Cc1c[nH]c(=O)[nH]c1=O</smiles>

(10) 2'-deoxyguanosine (11) Thymine<smiles>Nc1ncnc2[nH]cnc12</smiles>

(12) Adenine<smiles>Nc1ccn(C(=O)C2OC(CO)C(O)C2O)c(=O)n1</smiles>

(13) Cytidine

(14) Uracil<smiles>Nc1nc2[nH]cnc2c(=O)[nH]1</smiles>

(15) Guanine<smiles>C=C1NC(=O)c2nc[nH]c2N1</smiles><smiles>NCC(=O)O</smiles><smiles>NCCCC(=O)O</smiles><smiles>CC(C)CC(N)C(=O)O</smiles><smiles>CC(C)C(N)C(=O)O</smiles><smiles>CSCC[C@H](N)C(=O)O</smiles>

(16) Xanthine

(17) Glycine

(18) GABA

(19) Leucine

(20) Isoleucine

(21) Methionine<smiles>N[C@@H](Cc1ccccc1)C(=O)O</smiles>

(22) Phenylalanine<smiles>N[C@@H](Cc1c[nH]c2ccccc12)C(=O)O</smiles>

(23) Tryptophan<smiles>CC(N)C(=O)O</smiles>

(24) Alanine

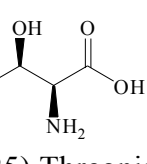

(25) Threonine

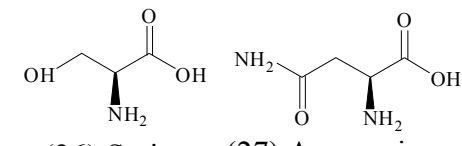

(26) Serine<smiles>CC(N)CCC(=O)O</smiles>

(28) Glutamine
(29) Glutamate

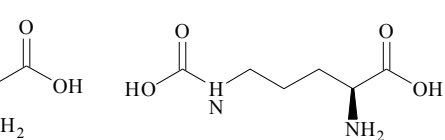

(30) Citrulline

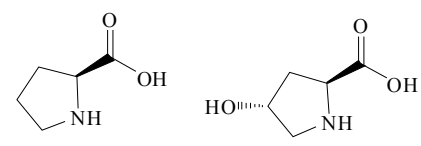

(31) Proline (32) Hydroxyproline

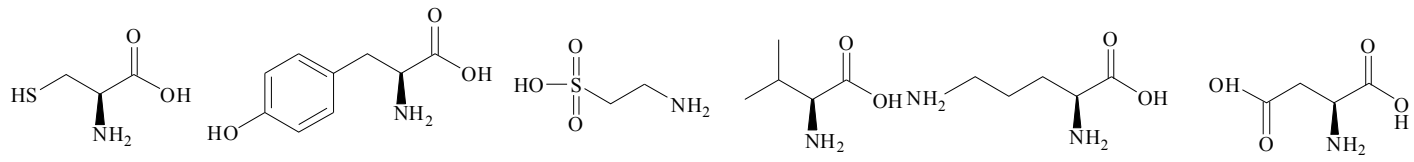

(33) Cysteine

(34) Tyrosine
(35) Taurine
(36) Valine (37) Ornithine

(38) Aspartate<smiles>NCCCCC(N)C(=O)O</smiles>

(39) Lysine

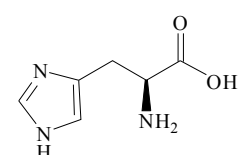

(40) Histidine<smiles>N=C(N)NCCCC(N)C(=O)O</smiles>

(41) Arginine

Figure 4. Chemical structures of the 41 identified amino acids nucleosides and nucleobases.

The S. nudus samples collected from the different habitats were identified by Prof. Ding Shaoxiong (Xiamen University, Fujian Province, China). After collection, the animals were kept in a $-80{ }^{\circ} \mathrm{C}$ refrigerator. Voucher specimens were deposited in Nanjing University of Chinese Medicine, China. After defrosting at the temperature of $4{ }^{\circ} \mathrm{C}$ and removing the excreta, the S. nudus were dissected into three parts, including body wall (SE), intestine (SI), coelomic fluid (SC) (Figure 5). 


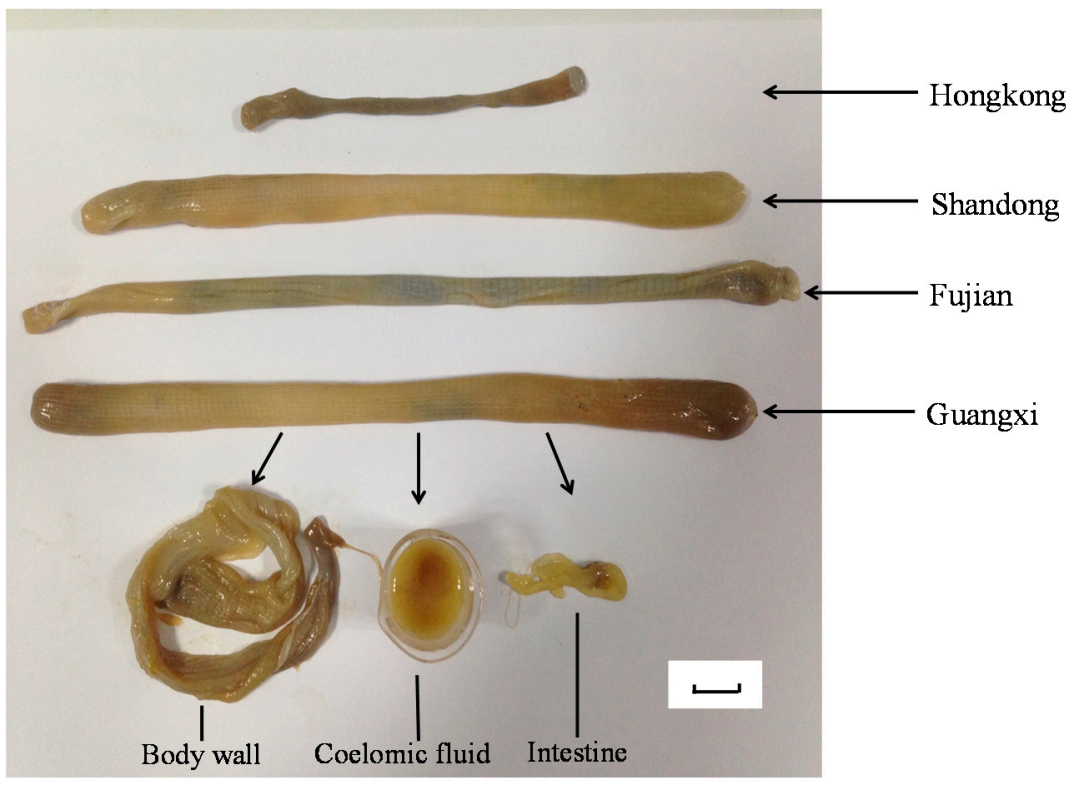

Figure 5. The appearance and anatomy of samples collected from four different habitats.

All three parts were freeze-dried in a vacuum freeze drier system (Labconco, Kansas City, MO, USA), then weighed and smashed, respectively. One gram of each dry sample was accurately weighed into a $50 \mathrm{~mL}$ conical flask, then $40 \mathrm{~mL}$ distilled water was added. All of the mixtures were placed into an ultrasonic bath $(40 \mathrm{kHz})$ for $60 \mathrm{~min}$ at room temperature. Meanwhile, the same samples were refluxed for $60 \mathrm{~min}$. Water was added to both sets oif samples to compensate for any lost during extraction. After centrifugation $(1,3000 \mathrm{r} / \mathrm{min})$ for $10 \mathrm{~min}$, the protein in the supernatants was removed by adding acetonitrile to double the volume and then stored at $4{ }^{\circ} \mathrm{C}$ and filtered through $0.22 \mu \mathrm{m}$ cellulose membrane filters prior to injection.

\subsection{UPLC-TQ-MS/MS Conditions}

The UPLC analysis was performed on a Waters ACQUITY UPLC H-class system (Waters, Milford, MA, USA) equipped with a quaternary pump solvent management system, an on-line degasser and an autosampler. The separation was performed on an ACQUITY UPLC BEH amide column $(2.1 \mathrm{~mm} \times 100 \mathrm{~mm}, 1.7 \mu \mathrm{m})$. The mobile phase was composed of $\mathrm{A}(5 \mathrm{mmol} / \mathrm{L}$ ammonium formate, $5 \mathrm{mmol} / \mathrm{L}$ ammonium acetate and $0.2 \%$ formic acid in aqueous solution) and B ( $1 \mathrm{mmol} / \mathrm{L}$ ammonium formate, $1 \mathrm{mmol} / \mathrm{L}$ ammonium acetate and $0.2 \%$ formic acid in acetonitrile) with a gradient elution: 0-3 min, $10 \%$ A; 3-9 min, $10 \%-18 \%$ A; $9-15 \mathrm{~min}, 18 \%-20 \%$ A, $15-16 \mathrm{~min}, 20 \%-46 \%$ A, $16-18 \mathrm{~min}$, $46 \% \mathrm{~A}$. The flow rate of the mobile phase was $0.4 \mathrm{~mL} / \mathrm{min}$, the injection volume was $2 \mu \mathrm{L}$, and the column temperature was maintained at $35^{\circ} \mathrm{C}$.

The mass spectrometry detection was performed by using a Xevo ${ }^{\mathrm{TM}}$ Triple Quadrupole MS (Waters Corp.) equipped with an electrospray ionisation (ESI) source operating in positive ionisation mode. The desolvation gas flow rate was set to $1000 \mathrm{~L} / \mathrm{h}$ at a temperature of $350{ }^{\circ} \mathrm{C}$, the cone gas flow rate was set at $20 \mathrm{~L} / \mathrm{h}$ and the source temperature was set at $120^{\circ} \mathrm{C}$. The capillary voltage was set to $3000 \mathrm{~V}$, the cone voltage and collision energy were set depending upon the MRM for each compound. Data were collected in MRM mode by screening parent and daughter ions simultaneously (Table 1). The raw data were acquired and processed with the Waters MassLynx 4.1 software (Waters China, Shanghai, China). 


\subsection{Data Processing}

The raw data were processed by the MassLynx 4.1 software, and the identification of free amino acids, nucleosides and nucleobases was carried out by comparing the retention time of target peaks with those of the standards, and the quantification was calculated by linear calibration plots of the peak. The statistical analysis was performed using the SPSS 17.0 software (SPSS, Chicago, IL, USA).

\subsection{Method Validation}

The linearity was verified by plotting the peak areas versus the corresponding concentrations of each analyte. The limit of detection (LOD) and limit of quantification (LOQ) were obtained by diluting the mixed standard working solution to the appropriate concentrations until the $\mathrm{S} / \mathrm{N}$ for each compound was about 3 and 10, respectively. The intra- and inter-day precisions were examined by analyzing the mixed standard solutions six times in a day and repeatedly in three consecutive days. To confirm the repeatability, six sample solutions from the same sample were prepared and analyzed in parallel. To evaluate the stability of the components, one of the sample solutions was analyzed in various periods $(0,2,4,6,12$, and $24 \mathrm{~h})$. Besides, there was also a recovery test was used to evaluate the accuracy of this method. It was performed by adding corresponding marker compounds with high $(120 \%)$, medium $(100 \%)$, and low $(80 \%)$ levels into accurately weighed samples, and then they were processed and analyzed with the same methods above. We prepared two groups of samples which were the standard solution and the matrix matching standard solution adding appropriate amounts of standards to the samples. Then we used the radios (the peak area of matrix matching standards/the peak area of standards) to investigate the matrix effects.

\section{Conclusions}

In this study, a simple, rapid and reliable UPLC-TQ-MS/MS method was developed and applied to simultaneously determine 25 free amino acids and 16 nucleosides and nucleobases in S. nudus extracts within $20 \mathrm{~min}$. The method had acceptable intra- and interday precision (RSD $<3.72 \%$, RSD $<3.42 \%$ ). The LODs and LOQs ranged from $0.003-0.229 \mu \mathrm{g} / \mathrm{mL}$ and $0.008-0.763 \mu \mathrm{g} / \mathrm{mL}$, respectively. Recoveries were between $94.03 \%$ and $106.33 \%$ with RSDs in the range of $0.64 \%-3.76 \%$ for all target compounds. Real sample data demonstrate that $S$. nudus is an excellent source of free amino acids, nucleosides and nucleobases with great nutritional and functional value. The contents of these compounds in different parts were significantly different, and in the order: (highest) coelomic fluid > body wall $>$ intestine (lowest). As the contents of xanthine, 2'-deoxyguanosine, taurine and glycine were the highest in each category, all of them could be proposed as markers for quality control of $S$. nudus. Moreover, the research results also provide a firm basis for further exploitation and development of $S$. nudus.

Acknowledgments: This work was supported by Marine Industry Research Special Funds for Public Welfare Projects (201205024-1) and A Project Funded by the Priority Academic Program Development of Jiangsu Higher Education Institutions (PAPD).

Author Contributions: Yahui Ge, Yuping Tang, and Jin-ao Duan conceptualized the project and Jin-ao Duan, Xiangzhi Lin, Ruirong Lin and Shaoxiong Ding headed the project. Yahui Ge, Yuping Tang, Sheng Guo, Xin Liu, Zhenhua Zhu, Lili Zhang and Pei Liu performed experiments. Yahui Ge, Yuping Tang, Sheng Guo were involved in writing the manuscript.

Conflicts of Interest: The authors declare no conflict of interest.

\section{References}

1. Pancucci-Papadopoulou, M.A.; Murina, G.V.V.; Zenetos, A. The Phylum Sipuncula in the Mediterranean Sea; Aghios Kosmas: Athens, Greece, 1999; p. 15.

2. State Administration of Traditional Chinese Medicine. Chinese Materia Medica; Shanghai Scientific and Technical Publishers: Shanghai, China, 2000; p. 7948.

3. Li, K.X.; Shen, X.R.; He, Y.; Jiang, D.W.; Liu, Y.M.; Hou, D.Y.; Chen, W. Effect of Sipunculus nudus polysaccharides on immunological function in mice. Chin. J. Mar. Drugs 2012, 31, 46-49. 
4. Zhang, G.H.; Zhao, M.M.; Wu, G.H. The nutritional components of enzymatic hydrolysate from Sipunculus nudus and effects on anti-oxidation. J. Food Sci. Biotechnol. 2007, 26, 80-84.

5. Chen, J.; Liu, Z.G.; Li, L.Y.; He, Y.C. Acute toxicity of three drugs acting on nymph of Sipunculus nudus. South China Fish. Sci. 2009, 5, 54-58.

6. Dong, L.F.; Zhang, Q.; Tong, T.; Xu, M.Z.; Chen, J.H. Amino acid composition of peanut worm Sipunculus nudus at different growth stages. South China Fish. Sci. 2012, 8, 60-65.

7. Zhang, G.H.; Zhao, M.M.; Wang, W. Isolation, Purification and Property Identification of Polysaccharide Contained in Sipunculus nudus. J. Food Sci. Biotechnol. 2006, 25, 63-66.

8. Hu, X.C. Some Trace Elements in Sipunculids. Fish. Sci. 2005, 24, 12-14.

9. Lei, D.Q.; Li, X.X.; Liao, G.S. Study of Fibrinolytic Enzyme from Sipunculus nudus Linnaeus in Guangxi Coastal Area. Nat. Prod. Res. Dev. 2013, 25, 897-902.

10. Guo, S.; Duan, J.A.; Qian, D.W.; Tang, Y.P.; Qian, Y.F.; Wu, D.W.; Su, S.L.; Shang, E.X. Rapid Determination of amino acids in fruits of Ziziphus jujube by hydrophilic interaction ultra-high-performance liquid chromatography coupled with triple-quadrupole mass spectrometry. J. Agric. Food Chem. 2013, 61, 2709-2719. [CrossRef] [PubMed]

11. Wu, L.M.; Chen, L.Z.; Selvaraj, J.N.; Wei, Y.; Wang, Y.; Li, Y.; Zhao, J.; Xue, X.F. Identification of the distribution of adenosine phosphates, nucleosides and nucleobases in royal jelly. Food Chem. 2015, 173, 1111-1118. [CrossRef] [PubMed]

12. Kong, W.J.; Jin, C.; Xiao, X.H.; Zhao, Y.L.; Liu, W.; Li, Z.L.; Zhang, P. Determination of multicomponent contents in Calculus bovis by ultra-performance liquid chromatography-evaporative light scattering detection and its application for quality control. J. Sep. Sci. 2010, 33, 1518-1527. [CrossRef] [PubMed]

13. Zhang, X.M.; Yang, F.Q.; Xia, Z.N. Recent advances in research on pharmaceutical functions of nucleosides and nucleotides in dietary foods. Food Sci. 2012, 33, 277-282.

14. Szterk, A.; Roszko, M. Simultaneous determination of free amino acids, L-Carnosine, purine, pyrimidine, and nucleosides in meat by liquid chromatography/single quadrupole mass spectrometry. J. Liq. Chromatogr. Relat. Technol. 2014, 37, 664-680. [CrossRef]

15. Chen, P.; Li, W.; Li, Q.; Wang, Y.H.; Li, Z.G.; Ni, Y.F. Identification and quantification of nucleosides and nucleobases in Geosaurus and Leech by hydrophilic-interaction chromatography. Talanta 2011, 85, 1634-1641. [CrossRef] [PubMed]

16. Cao, Y.; Duan, J.A.; Guo, J.M.; Guo, S. Rapid determination of nucleosides, nucleobases and free amino acids in brown seaweeds using ultra-performance liquid chromatography coupled with triple quadrupole mass spectrometry. J. Appl. Phycol. 2014, 26, 675-686. [CrossRef]

17. Yang, F.Q.; Zhang, X.M.; Ge, L.Y.; Tan, S.N. Determination of nucleosides and related compounds in Ganoderma. by CE-MS. J. China Pharm. Univ. 2011, 42, 337-341.

18. Cunico, R.L.; Schlabach, T. Comparison of ninhydrin and O-phthalaldehyde post-column detection techniques for high-performance liquid chromatography of free amino acids. J. Chromatogr. A 1983, 266, 461-470. [CrossRef]

19. Núñez, O.; Gallart-Ayala, H.; Martins, C.P.B.; Lucci, P. New trends in fast liquid chromatography for food and environmental analysis. J. Chromatogr. A 2012, 1228, 298-323. [CrossRef] [PubMed]

20. Koolen, H.H.F.; Silva, F.M.A.; Gozzo, F.C.; Souza, A.Q.L.; Souza, A.D.L. Antioxidant, antimicrobial activities and characterization of phenolic compounds from buriti (Mauritia flexuosa L.f.) by UPLC-ESI-MS/MS. Food Res. Int. 2013, 51, 467-473. [CrossRef]

21. Lerma-García, M.J.; Simó-Alfonso, E.F.; Méndez, A.; Liliberia, J.L.; Herrero-Martínez, J.M. Classification of extra virgin olive oils according to their genetic variety using linear discriminant analysis of sterol profiles established by ultra-performance liquid chromatography with mass spectrometry detection. Food Res. Int. 2011, 44, 103-108. [CrossRef]

22. Zhang, W.D.; Wang, P.Y.; Wang, Y.; Wang, Q.; Gu, Y.; Cao, J.; Wang, S.Q.; Wang, X.J. Simultaneous determination of aurantio-obtusin, chrysoobtusin, obtusin and 1-desmethylobtusin in rat plasma by UHPLC-MS/MS. Biomed. Chromatogr. 2014, 28, 369-374. [CrossRef] [PubMed]

23. Li, S.P.; Li, P.; Lai, C.M.; Gong, Y.X.; Kan Kelvin, K.W.; Dong Tina, T.X.; Tsim Karl, W.K.; Wang, Y.T. Simultaneous determination of ergosterol, nucleosides and their bases from natural and cultured Cordyceps by pressurized liquid extraction and high-performance liquid chromatography. J. Chromatogr. A 2004, 1036, 239-243. [CrossRef] [PubMed] 
24. Le Deun, E.; Van Der Werf, R.; Le Bail, G.; Le Quéré, J.M.; Guyot, S. HPLC-DAD-MS profiling of polyphenols responsible for the yellow-orange color in apple juices of different French cider apple varieties. J. Agric. Food Chem. 2015, 63, 7675-7684. [CrossRef] [PubMed]

25. Bataglion, G.A.; Silva, F.M.A.; Eberlin, M.N.; Koolen, H.H.F. Simultaneous quantification of phenolic compounds in buriti fruit (Mauritia flexuosa L.f.) by ultra-high performance liquid chromatography coupled to tendem mass spectrometry. Food Res. Int. 2014, 66, 396-400. [CrossRef]

26. Bajad, S.U.; Lu, W.; Kimball, E.H.; Yuan, J.; Peterson, C.; Rabinowitz, J.D. Separation and quantitation of water soluble cellular metabolites by hydrophilic interaction chromatography-tandem mass spectrometry. J. Chromatogr. A 2006, 1125, 76-78. [CrossRef] [PubMed]

27. Ge, Y.H.; Tang, Y.P.; Guo, S.; Liu, X.; Zhu, Z.H.; Liu, P.; Duan, J.A. Comparative analysis of amino acids, nucleosides, and nucleobases in Thais clavigera from different distribution regions by using hydrophilic interaction ultra-performance liquid chromatography coupled with triple quadrupole tandem mass spectrometry. Int. J. Anal. Chem. 2015. [CrossRef] [PubMed]

28. Ubhi, B.K.; Davenport, P.W.; Welch, M.; Riley, J.; Griffin, J.L.; Connor, S.C. Analysis of chloroformatederivatised amino acids, dipeptides and polyamines by LC-MS/MS. J. Chromatogr. B 2013, 934, 79-88. [CrossRef] [PubMed]

29. Guo, S.; Duan, J.A.; Qian, D.W.; Wang, H.Q.; Tang, Y.P.; Qian, Y.F.; Wu, D.W.; Su, S.L.; Shang, E.X. Hydrophilic interaction ultra-high performance liquid chromatography coupled with triple quadrupole mass spectrometry for determination of nucleotides, nucleosides and nucleobases in Ziziphus plants. J. Chromatogr. A 2013, 1301, 147-155. [CrossRef] [PubMed]

30. Zhou, G.S.; Pang, H.Q.; Tang, Y.P.; Yao, X.; Mo, X.; Zhu, S.Q.; Guo, S.; Qian, D.W.; Qian, Y.F.; Su, S.L.; et al Hydrophilic interaction ultra-performance liquid chromatography coupled with triple-quadrupole tandem mass spectrometry for highly rapid and sensitive analysis of underivatized amino acids in functional foods. Amino Acids 2013, 44, 1293-1305. [CrossRef] [PubMed]

31. Kasai, H. Analysis of a form of oxidative DNA damage, 8-hydroxy-2'-deoxyguanosine, as a marker of cellular oxidative stress during carcinogenesis. Mutat. Res. 1998, 387, 147-163. [CrossRef]

32. Alford, C.; Cox, H.; Wescott, R. The effect of Red Bull Energy Drink on human performance and mood. Amino Acids 2001, 21, 139-150. [CrossRef] [PubMed]

33. He, Y.; Shen, X.R.; Jiang, D.W.; Liu, Y.M.; Qian, T.T.; Mo, L.F. Effects of Sipunculus. nudus polysaccharides on learning and memory in memory obstruction model mice. Chin. J. Mar. Drug 2012, 31, 38-41.

34. Liu, Y.M.; Qian, T.T.; Mo, L.F.; He, Y.; Jiang, S.Q.; Shen, X.R. Study on the antifatigue effects of polysaccharides from Sipunculus nudus in mice. Chin. J. Mar. Drug 2012, 31, 41-44.

Sample Availability: Not available.

(C) 2016 by the authors; licensee MDPI, Basel, Switzerland. This article is an open access article distributed under the terms and conditions of the Creative Commons by Attribution (CC-BY) license (http:/ / creativecommons.org/licenses/by/4.0/). 